Pacific

Journal of

Mathematics

CONNECTED SUMS OF SELF-DUAL MANIFOLDS AND EQUIVARIANT RELATIVE SMOOTHINGS

Henrik Pedersen and Yat Sun Poon

Volume $191 \quad$ No. 1

November 1999 


\title{
CONNECTED SUMS OF SELF-DUAL MANIFOLDS AND EQUIVARIANT RELATIVE SMOOTHINGS
}

\author{
Henrik Pedersen and Yat Sun Poon
}

\begin{abstract}
In this paper we address a problem in differential geometry using tools from algebraic geometry and the theory of singular complex spaces. We obtain examples of compact four dimensional self-dual conformal manifolds with torus symmetry and positive scalar curvature from twistor spaces with divisors and we study the local moduli of such geometries.
\end{abstract}

\section{Introduction.}

Recall that a conformal 4-manifold is called self-dual if its Weyl curvature, considered as a bundle valued 2 -form, is in the +1 eigenspace of the Hodge star-operator [1]. Due to Schoen's proof [19] of the Yamabe conjecture it is known that within any conformal class on a compact manifold is a metric whose scalar curvature is constant and the sign of this constant is a conformal invariant. The main objective of this paper is to ensure that the scalar curvature is positive for the self-dual structures on the connected sums $n \mathbb{C P}^{2}$ of complex projective planes found in $[\mathbf{1 3}]$. The metrics admit a torus $T^{2}$ of orientation preserving conformal isometries.

For self-dual metrics the total space $Z$ of the bundle of anti-self-dual 2forms of unit length is a complex 3-manifold. This complex manifold is the twistor space [1] and it gives an alternative description of self-duality. Indeed, Donaldson and Friedman [2] used a desingularisation of a singular model of the desired twistor space to prove existence of self-dual structures on $n \mathbb{C P}^{2}$. The self-dual metric on $\mathbb{C P}^{2}$ is the Fubini-Study metric and the full moduli on $2 \mathbb{C P}^{2}$ had previously been obtained [15] via a different twistor construction. In [13] we adapted the theory of Donaldson and Friedman to obtain equivariant connected sums of compact self-dual manifolds.

If the symmetry group is at least three-dimensional it is known [16] that the conformal metric is of non-negative type. In contrast Kim [7] obtained $S^{1}$-symmetric examples of negative scalar curvature while LeBrun [11] gave examples on $n \mathbb{C P}^{2}$ of positive scalar curvature and with symmetry group $S^{1}$. These metrics were obtained via an ansatz involving monopoles on hyperbolic 3 -space. Similarly, Joyce [6] obtained $T^{2}$-symmetric metrics on $n \mathbb{C P}^{2}$ of positive type using hyperbolic monopoles in two dimensions. These constructions give relatively easy access to knowledge about scalar curvature 
while such insight is absent from our equivariant smoothings [13]. However, it is known [3] that if the complex manifold $Z$ carries effective divisors, then the corresponding metric is of non-negative type and if futhermore the intersection form of the 4-manifold is positive definite the scalar curvature is in fact positive [10].

Kim and Pontecorvo [8] extended the work of Donaldson and Friedman obtaining a way of constructing scalar-flat Kähler surfaces based on relative complex deformations of singular twistor spaces with divisors of degree 1 . We combine the equivariant and the relative smoothing programme to obtain a local moduli space of dimension $n-1$ of $T^{2}$-symmetric self-dual structures on $n \mathbb{C P}^{2}$ such that the associated twistor spaces all have smooth degree 1 and 2 divisors. In particular, all these self-dual metrics have positive scalar curvature.

We also use our equivariant relative smoothing approach to obtain antiself-dual Hermitian metrics with non-semi-free circle action on the blow up $\left(S^{1} \times S^{3}\right) \# n \overline{\mathbb{C P}}^{2}$, for $n \geq 3$, of the Hopf suface in more than two points. This should be compared with LeBrun's examples on the same spaces [12] but with semi-free circle symmetry.

\section{The Geometrical Construction.}

Our study of self-duality on a 4-manifold $M$ takes place on the associated twistor space $Z$. As a smooth manifold $Z$ is the total space of the sphere bundle $S\left(\Lambda_{-}^{2}\right) \rightarrow M$ of the bundle of anti-self-dual 2-forms. Equivalently, $Z$ is the total space of the bundle $P\left(V^{-}\right) \stackrel{\pi}{\longrightarrow} M$ of projectivised half spinors. The twistor space is a complex 3-manifold and for any point $x \in M$ the twistor line in $Z, \pi^{-1}(x)=L_{x}$, is a rational curve with normal bundle $N$ the sum $\mathcal{O}(1) \oplus \mathcal{O}(1)$ of degree 1 line bundles. Invariantly $N=H^{-} \otimes V_{x}^{+}$ where $H^{-} \rightarrow P\left(V_{x}^{-}\right)$is the hyperplane bundle. The antipodal map on the fibers of $\pi: Z \rightarrow M$ induces a real structure on $Z$ [1]. The anti-canonical bundle $K^{-1}=\Lambda^{3,0} \mathrm{~T} Z$ of $Z$ has a square root $K^{-\frac{1}{2}}$ which, when restricted onto a twistor line, coincides with the degree 2 holomorphic line bundle $\mathcal{O}(2)$. The zero set in the twistor space of a real holomorphic section of $K^{-\frac{1}{2}}$ is called a degree 2 divisor. Such a divisor $S$ may either be irreducible, in which case it is smooth [14], or it may decompose into a conjugate pair $D, \bar{D}$ of smooth degree 1 divisors meeting in exactly one twistor line [17].

Now, assume we have two self-dual manifolds $M_{1}$ and $M_{2}$ with associated twistor spaces $Z_{1}$ and $Z_{2}$. Furthermore, assume $Z_{1}$ contains an irreducible degree 2 divisor $S$ and let $L_{1}=L_{x_{1}}$ be a twistor line above $x_{1} \in M_{1}$ meeting $S$ transversely in a conjugate pair of points $q$ and $\bar{q}$. Also, let $D_{2}, \overline{D_{2}}$ be a conjugate pair of divisors in $Z_{2}$ meeting in the twistor line $L_{2}=L_{x_{2}}$ above $x_{2} \in M_{2}$. Then, following Donaldson and Friedman [2], we blow up the twistor spaces $Z_{i}$ along the twistor lines $L_{i}, i=1,2$. The proper transform 
$\tilde{S}$ of $S$ in the blow-up $\tilde{Z}_{1}$ of $Z_{1}$ meets the exceptional divisor $Q_{1}$ in two rational curves $C_{1}$ and $\bar{C}_{1}$. The normal bundles $N_{C_{1}}^{\tilde{S}}, N_{\bar{C}_{1}}^{\tilde{S}}$ relative to $\tilde{S}$ are both equal to $\mathcal{O}(-1)$. In the blow-up $\tilde{Z}_{2}$ of $Z_{2}$ the proper transforms $\tilde{D}_{2}, \tilde{\bar{D}}_{2}$ of $D_{2}, \bar{D}_{2}$, respectively, meet the exceptional divisor $Q_{2}$ in a conjugate pair of rational curves $C_{2}, \bar{C}_{2}$ with normal bundles $N_{C_{2}}^{\tilde{D}_{2}}=\mathcal{O}(1)$ and $N_{\bar{C}_{2}}^{\tilde{\bar{D}}_{2}}=\mathcal{O}(1)$.

From an orientation reversing isometry $\phi: T_{x_{1}} M_{1} \rightarrow T_{x_{2}} M_{2}$ we obtain a holomorphic isomorphism

$$
\phi=\phi_{+} \times \phi_{-}: Q_{1}=P\left(V_{x_{1}}^{+}\right) \times P\left(V_{x_{1}}^{-}\right) \rightarrow Q_{2}=P\left(V_{x_{2}}^{-}\right) \times P\left(V_{x_{2}}^{+}\right)
$$

and we construct the singular complex space $\mathcal{Z}=\tilde{Z}_{1} \cup_{\phi} \tilde{Z}_{2}$ with normal crossing singularity along $Q=Q_{1}=\phi^{-1}\left(Q_{2}\right)$. In $\mathcal{Z}$ we have the singular divisor $\mathcal{S}$ with normal crossing singularities along $C=C_{1}=\phi^{-1}\left(C_{2}\right)$ and $\bar{C}=\bar{C}_{1}=\phi^{-1}\left(\bar{C}_{2}\right)$.

Inspired by Kim and Pontecorvo [8] we now proceed to study smoothings of the pair $(\mathcal{Z}, \mathcal{S})$. The aim is to show that in some cases the connected sum has a twistor space with a degree 2 divisor. Let us introduce the necessary notation: If $Y_{1}, \ldots, Y_{n}$ are smooth submanifolds of a compact complex manifold $X$ we consider the sheaf $\Theta_{X Y_{1}, \ldots, Y_{n}}$ of holomorphic vector fields on $X$ which are tangent to $Y_{i}$ along $Y_{i}, i=1, \ldots, n$. It is well known [9] that infinitesimal relative deformations are given by the first cohomology of this sheaf and that obstructions lie in the second cohomology. For a compact singular complex space $\mathcal{X}$ the deformation theory is described in terms of global extension groups $T_{\mathcal{X}}^{i}=\operatorname{Ext}^{i}\left(\Omega_{\mathcal{X}}, \mathcal{O}_{\mathcal{X}}\right)$ where $\Omega_{\mathcal{X}}$ is the sheaf of Kähler differentials and $\mathcal{O}_{\mathcal{X}}$ is the structure sheaf. These groups are computed from the sheaves $\tau_{\mathcal{X}}^{i}=\mathcal{E} x t^{i}\left(\Omega_{\mathcal{X}}, \mathcal{O}_{\mathcal{X}}\right)$ using the local to global spectral sequence $E_{2}^{p, q}=H^{p}\left(\tau_{\mathcal{X}}^{q}\right) \Rightarrow T_{\mathcal{X}}^{p+q}[2],[4]$. Here $T_{\mathcal{X}}^{0}$ is the Lie algebra of the group of automorphisms of $\mathcal{X}$, the first order deformations lie in $T_{\mathcal{X}}^{1}$ and obstructions are in $T_{\mathcal{X}}^{2}$. For normal crossings $\tau_{\mathcal{X}}^{2}=0$ and the local to global spectral sequence is given as

$$
0 \rightarrow H^{1}\left(\tau_{\mathcal{X}}^{0}\right) \rightarrow T_{\mathcal{X}}^{1} \rightarrow H^{0}\left(\tau_{\mathcal{X}}^{1}\right) \rightarrow H^{2}\left(\tau_{\mathcal{X}}^{0}\right) \rightarrow T_{\mathcal{X}}^{2} \rightarrow H^{1}\left(\tau_{\mathcal{X}}^{1}\right) .
$$

In the situations we are going to investigate, we have $H^{1}\left(\tau_{\mathcal{X}}^{1}\right)=0$. Furthermore, we impose conditions implying the vanishing of $H^{2}\left(\tau_{\mathcal{X}}^{0}\right)$ so that $T_{\mathcal{X}}^{2}=0$ and the deformations are unobstructed and parametrised by $T_{\mathcal{X}}^{1}$. The subspace $H^{1}\left(\tau_{\mathcal{X}}^{0}\right)$ corresponds to deformations for which the singularities remain locally a product. If the image of an element in the projection $T_{\mathcal{X}}^{1} \rightarrow H^{0}\left(\tau_{\mathcal{X}}^{1}\right)$ does not vanish the corresponding deformed space is smooth. Furthermore, for $\mathcal{X}$ equal to the singular twistor space $\mathcal{Z}$ the smoothing results in a twistor space with real structure [2].

The singular theory above will be applied to $\mathcal{X}=\mathcal{Z}$ and $\mathcal{X}=\mathcal{S}$. To study deformations of the singular pair $f: \mathcal{S} \hookrightarrow \mathcal{Z}$ we employ the theory of Ran [18]: Let $T_{\mathcal{Z} \mid \mathcal{S}}^{i}$ denote the extension groups $\operatorname{Ext}^{i}\left(\Omega_{\mathcal{Z} \mid \mathcal{S}}, \mathcal{O}_{\mathcal{S}}\right)[\mathbf{8}]$ with the local 
to global spectral sequence $E_{2}^{p, q}=H^{p}\left(\mathcal{E} x t^{q}\left(\Omega_{\mathcal{Z} \mid \mathcal{S}}, \mathcal{O}_{\mathcal{S}}\right)\right)$ where $E_{2}^{p, q} \Rightarrow T_{\mathcal{Z} \mid \mathcal{S}}^{p+q}$. This gives the sequence

$$
0 \rightarrow H^{1}\left(\tau_{\mathcal{Z} \mid \mathcal{S}}^{0}\right) \rightarrow T_{\mathcal{Z} \mid \mathcal{S}}^{1} \rightarrow H^{0}\left(\tau_{\mathcal{Z} \mid \mathcal{S}}^{1}\right) \rightarrow H^{2}\left(\tau_{\mathcal{Z} \mid \mathcal{S}}^{0}\right)
$$

Furthermore, the groups $T_{\mathcal{Z} \mid \mathcal{S}}^{i}$ fit into a sequence

$$
\rightarrow T_{f}^{1} \rightarrow T_{\mathcal{S}}^{1} \oplus T_{\mathcal{Z}}^{1} \rightarrow T_{\mathcal{Z} \mid \mathcal{S}}^{1} \rightarrow T_{f}^{2} \rightarrow T_{\mathcal{S}}^{2} \oplus T_{\mathcal{Z}}^{2} \rightarrow
$$

where $T_{f}^{i}$ is a natural derived functor and $T_{f}^{1}$ gives infinitesimal deformations of the singular pair. Also, $T_{f}^{0}$ is the Lie algebra of the symmetries of the pair $(\mathcal{Z}, \mathcal{S})$ and $T_{f}^{2}$ is the obstruction space. For pairs $(\mathcal{X}, \mathcal{Y})$ of complex spaces we also have the sheaf $\tau_{\mathcal{X} Y}^{0}$ of derivations of $\mathcal{X}$ preserving the ideal sheaf of $\mathcal{Y}$. This sheaf of relative derivations coincide with $\Theta_{X Y}$ for pairs of smooth manifolds. From inclusions and restrictions we obtain the exact sequence

$$
0 \rightarrow \tau_{\mathcal{X} Y}^{0} \rightarrow \tau_{\mathcal{Y}}^{0} \oplus \tau_{\mathcal{X}}^{0} \rightarrow \tau_{\mathcal{X} \mid \mathcal{Y}}^{0} \rightarrow 0
$$

In the associated sequence on cohomology

$$
H^{1}\left(\tau_{\mathcal{X} \mathcal{Y}}^{0}\right) \rightarrow H^{1}\left(\tau_{\mathcal{Y}}^{0}\right) \oplus H^{1}\left(\tau_{\mathcal{X}}^{0}\right) \rightarrow H^{1}\left(\tau_{\mathcal{X} \mid \mathcal{Y}}^{0}\right) \rightarrow H^{2}\left(\tau_{\mathcal{X} \mathcal{Y}}^{0}\right)
$$

the first term corresponds to deformations for which the singularities remain locally a product so $H^{1}\left(\tau_{\mathcal{X} Y}^{0}\right)$ sits naturally inside $T_{f}^{1}$. Call the quotient space $\Delta$. Then we have the short exact sequence

$$
0 \rightarrow H^{1}\left(\tau_{\mathcal{X} Y}^{0}\right) \rightarrow T_{f}^{1} \rightarrow \Delta \rightarrow 0 .
$$

Now put together (2.1) with $\mathcal{X}=\mathcal{Z}$ and $\mathcal{X}=\mathcal{S},(2.2)$, (2.3), (2.5) with $\mathcal{X}=\mathcal{Z}$ and $\mathcal{Y}=\mathcal{S}$ and (2.6) to get the following commutative diagram.

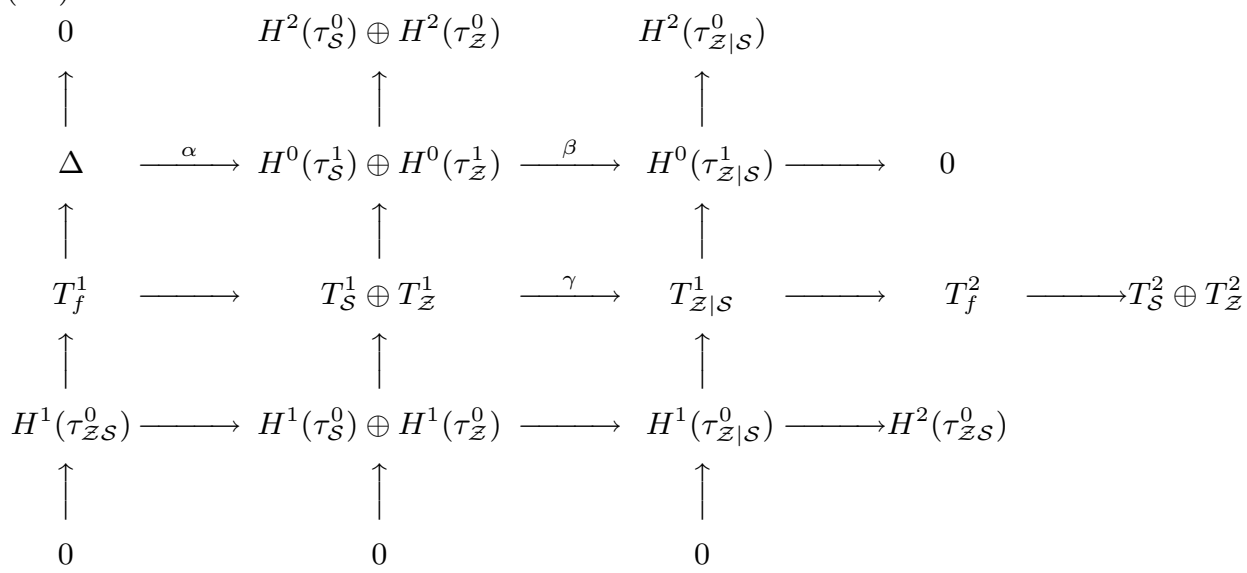

The morphism $\alpha$ is the quotient of the morphism $T_{f}^{1} \rightarrow T_{\mathcal{S}}^{1} \oplus T_{\mathcal{Z}}^{1}$ and the squares in the diagram are commutative by naturality. The row with the morphisms $\alpha$ and $\beta$ needs more explanation: The sheaf $\tau_{\mathcal{Z}}^{1}$ is supported on 
$Q \subseteq \mathcal{Z}$ and is the sheaf of sections of $N_{Q_{1}}^{\tilde{Z}_{1}} \otimes \phi^{*} N_{Q_{2}}^{\tilde{Z}_{2}}=\mathcal{O}_{Q}[\mathbf{2}]$. Likewise, a choice of trivialization $\tau_{\mathcal{Z}}^{1} \cong \mathcal{O}_{Q}$ induces trivializations $\tau_{\mathcal{S}}^{1} \cong \mathcal{O}_{C \bar{C}}$ and $\tau_{\mathcal{Z} \mid \mathcal{S}}^{1} \cong$ $\mathcal{O}_{Q \mid \mathcal{S}} \cong \mathcal{O}_{C \bar{C}}$. Then $H^{0}\left(\tau_{\mathcal{S}}^{1}\right)=H^{0}\left(\mathcal{O}_{C}\right) \oplus H^{0}\left(\mathcal{O}_{\bar{C}}\right), H^{0}\left(\tau_{\mathcal{Z}}^{1}\right)=H^{0}\left(\mathcal{O}_{Q}\right)$, $H^{0}\left(\tau_{\mathcal{Z} \mid \mathcal{S}}^{1}\right)=H^{0}\left(\mathcal{O}_{C}\right) \oplus H^{0}\left(\mathcal{O}_{\bar{C}}\right)$ and $\beta((a, b), c)=(a-c, b-c)$ which certainly is surjective. The kernel of $\beta$ is $\langle c, c, c\rangle \cong \mathbb{C}$.

Now we shall make the following assumptions which are satisfied in examples to be considered later:

Assumption 2.1. In the case where the degree 2 divisor is irreducible we assume the vanishing of the following cohomology groups: $H^{2}\left(Z_{i}, \Theta_{Z_{i}}\right)$, $i=1,2 ; \quad H^{2}\left(S, \Theta_{S}\right) ; \quad H^{2}\left(D_{2}, \Theta_{D_{2}}\right) ; \quad H^{2}\left(\bar{D}_{2}, \Theta_{\bar{D}_{2}}\right) ; \quad H^{2}\left(Z_{1}, \Theta_{Z_{1} S}\right)$ and $H^{2}\left(Z_{2}, \Theta_{Z_{2} D_{2} \bar{D}_{2}}\right)$.

We shall show how these assumptions lead to the vanishing of $H^{2}\left(\tau_{\mathcal{S}}^{0}\right)$, $H^{2}\left(\tau_{\mathcal{Z}}^{0}\right)$ and $H^{2}\left(\tau_{\mathcal{Z} \mathcal{S}}^{0}\right)$ and therefore from $(2.1)$ we have the vanishing of the obstruction spaces $T_{\mathcal{Z}}^{2}$ and $T_{\mathcal{S}}^{2}$. We also see that $\Delta$ is equal to the kernel of $\beta$. Now a diagram chasing gives the surjectivity of the morphism $\gamma$ so $T_{f}^{2}=0$. Therefore, any element $\omega$ in the complement of the hyperplane $H^{1}\left(\tau_{\mathcal{Z} S}^{0}\right)$ of $T_{f}^{1}$ gives smoothings of $\mathcal{Z}$ and $\mathcal{S}$ as $\omega$ maps to non-zero elements in $H^{0}\left(\tau_{\mathcal{S}}^{1}\right)$ and $H^{0}\left(\tau_{\mathcal{Z}}^{1}\right)$. Thus the twistor space of the connected sum $M_{1} \# M_{2}$ has a degree 2 divisor. Later we return to a calculation of the dimension of the space $T_{f}^{1}$ of infinitesimal deformations.

We shall also consider the situation where the degree 2 divisor $S$ decomposes into a conjugate pair $D_{1}, \bar{D}_{1}$ of degree 1 divisors meeting in a twistor line $L$. We still blow up $Z_{1}$ along a twistor line $L_{1}$ intersecting $D_{1}+\bar{D}_{1}$ transversely in a conjugate pair of points. Also the proper transforms $\tilde{D}_{1}, \tilde{\bar{D}}_{1}$ meet $Q_{1}$ in $C_{1}$ and $\bar{C}_{1}$. As before we construct the singular twistor space $\mathcal{Z}$ with the singular divisors $\mathcal{D}, \overline{\mathcal{D}}$ having normal crossing singularities along $C$ and $\bar{C}$ respectively. The divisors $\mathcal{D}$ and $\overline{\mathcal{D}}$ meet in $L$. In this situation $\mathcal{S}$ is the singular space $\mathcal{D} \cup_{L} \overline{\mathcal{D}}$ with normal crossing singularities along $L, C$ and $\bar{C}$. We make the

Assumption 2.2. In the case where the degree 2 divisor is reducible we assume the vanishing of the following cohomology groups: $H^{2}\left(Z_{i}, \Theta_{Z_{i}}\right)$, $H^{2}\left(D_{i}, \Theta_{D_{i}}\right), H^{2}\left(\bar{D}_{i}, \Theta_{\bar{D}_{i}}\right), i=1,2 ; H^{2}\left(Z_{1}, \Theta_{Z_{1} S}\right)$ and $H^{2}\left(Z_{2}, \Theta_{Z_{2} D_{2} \bar{D}_{2}}\right)$.

Furthermore, we get a diagram similar to (2.7) and as in the case where $S$ is irreducible the smoothing is unobstructed. However, we do not control the singularities of $\mathcal{S}$ along $L$. Therefore, a smoothing of $\mathcal{Z}$ may produce an irreducible smooth degree 2 divisor. We shall discuss this problem at the end of Section 5 . 


\section{Symmetries.}

In this section we assume $M_{1}$ and $M_{2}$ have a torus group $T^{2}$ of orientation preserving conformal transformations with fixed points $x_{1}$ and $x_{2}$ respectively.

Furthermore, the orientation reversing isometry $\phi: T_{x_{1}} M_{1} \rightarrow T_{x_{2}} M_{2}$, used to make the connected sum, is assumed to be an equivariant map. In this situation we proved in [13] that if the smoothing is unobstructed then also the equivariant smoothing is unobstructed. We refer to that paper for details. Now we want to impose the symmetries onto the relative smoothing described in Section 2.

Recall [13] that we may choose metrics in $M_{1}$ and $M_{2}$ for which the torus acts as isometries. Therefore, the isotropy representation $\iota$ at the fixed points map into $S O(4)$. Also, the symmetries lift to the twistor space to give real holomorphic automorphisms. If $x_{1}$ is a fixed point in $M_{1}$, and $L_{1}$ is the twistor line with normal bundle $N$, then the well known isomorphisms from twistor theory

$$
\left(T_{x_{1}} M_{1}\right)^{\mathbb{C}}=H^{0}\left(L_{1}, N\right)=V_{x_{1}}^{+} \otimes V_{x_{1}}^{-}
$$

are equivariant. This means the isotropy representation on the complexified tangent space coincide with the induced holomorphic action on the sections of the normal bundle and with the tensor product $\iota_{+} \otimes \iota_{-}$on $V_{x_{1}}^{+} \otimes V_{x_{1}}^{-}$. Here $\iota_{ \pm}$are defined modulo $\mathbb{Z}_{2}$ from the isotropy representation $\iota$ and the projections onto the factors of $S U(2) \times S U(2)$.

Since the twistor line $L_{1}$ intersects the divisor $S$ transversely at two distinct points, we assume that $S$ is reducible to $D_{1}+\bar{D}_{1}$ when we consider the twistor geometry in a neighbourhood of $L_{1}$. Now suppose the divisors $D_{i}, \bar{D}_{i}$ in $Z_{i}, i=1,2$ are invariant. Then the points $q=P\left(V_{x_{1}}^{-}\right) \cap D_{1}$ and $\bar{q}=P\left(V_{x_{1}}^{-}\right) \cap \bar{D}_{1}$ are fixed by the action on $Z_{1}$. Therefore if $v \in V_{x_{1}}^{-}$represents $q$ we have $V_{x_{1}}^{-}=\operatorname{span}(v) \oplus \operatorname{span}(\bar{v})$ as a decomposition into $T^{2}$-invariant subspaces. Furthermore, from the definition of the complex structure on $Z_{1}$ the horizontal space $T_{q} D_{1}$ is equal to $V_{x_{1}}^{+} \otimes(\operatorname{span}(v))^{*}$ with the action $\iota_{+} \otimes \iota_{-}^{*}$. We have $C_{1}=P\left(T_{q} D_{1}\right)$ and the normal bundle $N_{C_{1}}^{\tilde{D}_{1}}$ is the universal subbundle of $T_{q} D_{1}$ over $C_{1}$.

On the other side we have $L_{2}=P\left(V_{x_{2}}^{-}\right)$and the normal bundles $N_{C_{2}}^{\tilde{D}_{2}}=$ $N_{L_{2}}^{D_{2}}, N_{\bar{C}_{2}}^{\tilde{\bar{D}}_{2}}=N_{L_{2}}^{\bar{D}_{2}}$. As $L_{2}$ is equal to the transversal intersection $D_{2} \cap \bar{D}_{2}$ we have $N_{L_{2}}^{Z_{2}}=N_{L_{2}}^{D_{2}} \oplus N_{L_{2}}^{\bar{D}_{2}}$ and this is a decomposition into invariant subbundles. From twistor theory we have $N_{L_{2}}^{Z_{2}}=V_{x_{2}}^{+} \otimes H^{-}$. Also, the torus action on $V_{x_{2}}^{+}$decomposes, $V_{x_{2}}^{+}=E \oplus \bar{E}$, and the only decomposition of $V_{x_{2}}^{+} \otimes H^{-}$into invariant subspaces is $\left(E \otimes H^{-}\right) \oplus\left(\bar{E} \otimes H^{-}\right)$. We may therefore assume that $N_{L_{2}}^{D_{2}}=E \otimes H^{-}$and $N_{L_{2}}^{\bar{D}_{2}}=\bar{E} \otimes H^{-}$. 
The $T^{2}$-equivariant isomorphism $\phi_{-}: V_{x_{1}}^{-} \rightarrow V_{x_{2}}^{+}$induced by $\phi: T_{x_{1}} M_{1} \rightarrow$ $T_{x_{2}} M_{2}$ can be assumed to satisfy $\phi_{-}(\operatorname{span}(v))=E$ and $\phi_{-}(\operatorname{span}(\bar{v}))=\bar{E}$. Now we have $\mathcal{D}=\tilde{D}_{1} \cup_{\phi_{+}} \tilde{D}_{2}$ where

$$
\phi_{+}: C_{1}=P\left(T_{q} D_{1}\right)=P\left(V_{x_{1}}^{+}\right) \rightarrow P\left(V_{x_{2}}^{-}\right)=C_{2}
$$

and similarly with $\overline{\mathcal{D}}$. Recall the fact $[\mathbf{1 3}]$ that $H^{0}\left(\tau_{\mathcal{Z}}^{1}\right)_{T^{2}}=H^{0}\left(\tau_{\mathcal{Z}}^{1}\right) \cong \mathbb{C}$ which is true because $\tau_{\mathcal{Z}}^{1}=N_{Q_{1}}^{\tilde{Z}_{1}} \otimes \phi^{*}\left(N_{Q_{2}}^{\tilde{Z}_{2}}\right)=\mathcal{O}_{Q}$ and the action is trivial. Also, $H^{0}\left(\tau_{\mathcal{Z} \mid \mathcal{S}}^{1}\right)_{T^{2}}=H^{0}\left(\tau_{\mathcal{Z} \mid \mathcal{S}}^{1}\right) \cong \mathbb{C}^{2}$ because $\tau_{\mathcal{Z}}^{1}$ is supported by $Q$ and $Q$ intersects $\mathcal{S}$ transversely along $C$ and $\bar{C}$.

Likewise we shall need the following:

Lemma 3.1. The sheaf $\tau_{\mathcal{D}}^{1}$ is supported on the curve $C$ and is trivial. All the sections are $T^{2}$-invariant, i.e. $H^{0}\left(\tau_{\mathcal{D}}^{1}\right)_{T^{2}}=H^{0}\left(\tau_{\mathcal{D}}^{1}\right)=\mathbb{C}$. Similarly $H^{0}\left(\tau_{\mathcal{S}}^{1}\right)_{T^{2}}=H^{0}\left(\tau_{\mathcal{S}}^{1}\right)=\mathbb{C}^{2}$ when the degree 2 divisor $S$ is irreducible.

Proof. Since the singularity is a normal crossing we have $\tau_{\mathcal{D}}^{1}=N_{C_{1}}^{\tilde{D}_{1}} \otimes \phi^{*} N_{C_{2}}^{\tilde{D}_{2}}$ [2]. The curve $C_{1}$ is the exceptional divisor of the blowing up of $q$ in $D_{1}$ so $N_{C_{1}}^{\tilde{D}_{1}}=\mathcal{O}(-1)$. Also, $N_{C_{2}}^{\tilde{D}_{2}}$ is isomorphic to $N_{L_{2}}^{D_{2}}$ so $N_{C_{2}}^{\tilde{D}_{2}}=\mathcal{O}(1)$. Thus $\tau_{\mathcal{D}}^{1}=\mathcal{O}_{C}$. Furthermore, the torus action is trivial on $\tau_{\mathcal{D}}^{1}$ : the action on $N_{C_{1}}^{\tilde{D}_{1}}$ is $\iota_{+} \otimes \iota_{-}^{*}$ as this normal bundle is the universal subbundle of $T_{q} D_{1}=$ $V_{x_{1}}^{+} \otimes(\operatorname{span}(v))^{*}$. On the other hand $\phi^{*} N_{C_{2}}^{\tilde{D}_{2}}=\phi^{*} N_{L_{2}}^{D_{2}}=\phi^{*}\left(E \otimes H^{-}\right)=$ $\left(\phi_{-}^{*} E\right) \otimes\left(\phi_{+}^{*} H^{-}\right)=\operatorname{span}(v) \otimes H^{+}=\left((\operatorname{span}(v))^{*} \otimes U^{+}\right)^{*}=\left(N_{C_{1}}^{\tilde{D}_{1}}\right)^{*}$. The torus action on $N_{L_{2}}^{D_{2}}=E \otimes H^{-}$is $\iota_{+} \otimes \iota_{-}^{*}$. Therefore the action on $\phi^{*} N_{L_{2}}^{D_{2}}$ is $\iota_{-} \otimes \iota_{+}^{*}$ and we get the trivial action on $\tau_{\mathcal{D}}^{1}=N_{C_{1}}^{\tilde{D}_{1}} \otimes \phi^{*} N_{C_{2}}^{\tilde{D}_{2}}$ from the tensor product $\iota_{+} \otimes \iota_{-}^{*} \otimes \iota_{-} \otimes \iota_{+}^{*}$.

\section{Vanishing Theorems and the Unobstructed Case.}

We consider an irreducible degree 2 divisor $S$ and address the problem of proving the vanishing of $H^{2}\left(\tau_{\mathcal{Z}}^{0}\right), H^{2}\left(\tau_{\mathcal{S}}^{0}\right)$ and $H^{2}\left(\tau_{\mathcal{Z} \mathcal{S}}^{0}\right)$. This will lead to a theorem on the unobstructed $T^{2}$-equivariant relative smoothing.

First some technical lemmae:

Lemma 4.1. Let $Y \subseteq X$ be a smooth hypersurface in a complex manifold $X$. Then for any sufficiently small open set $U \subseteq X$ we have $H^{i}\left(U, \Theta_{X}\right)=0$ and $H^{i}\left(U, \Theta_{X Y}\right)=0, i \geq 1$.

Proof. We have $H^{i}\left(U, \Theta_{X}\right)=0, i \geq 1$, as $\Theta_{X}$ is locally free and $H^{i}(U, \mathcal{O})=0$ by the $\bar{\partial}$-Poincaré Lemma. Similarly $H^{i}\left(U \cap Y, \mathcal{O}_{Y}\left(N_{Y}^{X}\right)\right)=0, i \geq 1$.

From the sequence

$$
0 \rightarrow \Theta_{X Y} \rightarrow \Theta_{X} \rightarrow \mathcal{O}_{Y}\left(N_{X}^{Y}\right) \rightarrow 0
$$


we get $H^{i}\left(U, \Theta_{X Y}\right)=0, i \geq 2$. We may assume $U$ is a coordinate patch where the vanishing $x_{1}=0$ of the first coordinate defines $Y$. Therefore

$$
0 \rightarrow H^{0}\left(U, \Theta_{X Y}\right) \rightarrow H^{0}\left(U, \Theta_{X}\right) \rightarrow H^{0}\left(U \cap Y, \mathcal{O}_{Y}\left(N_{Y}^{X}\right)\right) \rightarrow 0
$$

is exact and it follows that $H^{1}\left(U, \Theta_{X Y}\right)=0$.

Lemma 4.2. Let $b_{i}: \tilde{Z}_{i} \rightarrow Z_{i}$ be the blow-up of $Z_{i}$ along the real twistor line $L_{i}, i=1,2$. Then for all $j \geq 0: H^{j}\left(\tilde{Z}_{i}, \Theta_{\tilde{Z}_{i}}\right)=H^{j}\left(Z_{i}, \Theta_{Z_{i} L_{i}}\right)$.

Proof. We drop the subscript " $i$ ". The direct image sheaf $b_{*} \Theta_{\tilde{Z}}$ is isomorphic to $\Theta_{Z L}$ via the differential of $b$

$$
d b: H^{0}\left(b^{-1}(U), \Theta_{\tilde{Z}}\right) \rightarrow H^{0}\left(U, \Theta_{Z L}\right)
$$

for $U \subseteq Z$ open. Certainly $d b$ maps into $H^{0}\left(U, \Theta_{Z L}\right)$ if we can prove that any vector field on $b^{-1}(U)$ is tangential to $Q$ along $Q$. But this follows from the vanishing of $H^{0}(Q,[Q])=H^{0}\left(\mathbb{C P}_{1} \times \mathbb{C P}_{1}, \mathcal{O}(1,-1)\right)$ and from the sequence

$$
\left.\left.0 \rightarrow T Q \rightarrow T \tilde{Z}\right|_{Q} \rightarrow[Q]\right|_{Q} \rightarrow 0 .
$$

Conversely, let $L$ be given in local coordinates $\left(z_{1}, z_{2}, z_{3}\right)$ by $z_{2}=z_{3}=0$. Then, a vector field $X=a_{1} \frac{\partial}{\partial z_{1}}+\left(a_{2} z_{2}+a_{3} z_{3}\right) \frac{\partial}{\partial z_{2}}+\left(a_{4} z_{2}+a_{5} z_{3}\right) \frac{\partial}{\partial z_{3}}$ in $H^{0}\left(U, \Theta_{Z L}\right)$ can be lifted to $\tilde{X}=a_{1} \frac{\partial}{\partial z_{1}}+\left(a_{2} z_{2}+a_{3} z_{2} \ell\right) \frac{\partial}{\partial z_{2}}+\left(a_{4}+a_{5} \ell\right) \frac{\partial}{\partial \ell}$ in $H^{0}\left(b^{-1}(U), \Theta_{\tilde{Z}}\right)$ where $\ell=\frac{\ell_{3}}{\ell_{2}}$ is a coordinate on $b^{-1}(U)=\left\{\left(\left(z_{1}, z_{2}, z_{3}\right)\right.\right.$, $\left.\left.\left[\ell_{2}, \ell_{3}\right]\right) \in U \times \mathbb{C P}^{1} \mid \ell_{2} z_{3}=\ell_{3} z_{2}\right\}$.

The lemma now follows from the Leray spectral sequence [4] if we can prove that the sheaf $R^{i} b_{*}\left(\Theta_{\tilde{Z}}\right)$ vanishes for all $i \geq 1$ where $R^{i} b_{*}\left(\Theta_{\tilde{Z}}\right)(U)=$ $H^{i}\left(b^{-1}(U), \Theta_{\tilde{Z}}\right)$. Thus, consider the Leray cover $V_{1} \cup V_{2}$ of $b^{-1}(U)$ given by coordinates $\left(z_{1}, z_{2}, \ell\right)$ and $\left(z_{1}, z_{3}, \frac{1}{\ell}\right)$ respectively. Then $H^{i}\left(b^{-1}(U), \Theta_{\tilde{Z}}\right)=0$, $i \geq 2$, and $H^{1}\left(b^{-1}(U), \Theta_{\tilde{Z}}\right)=H^{1}\left(V_{1} \cap V_{2}, \Theta_{\tilde{Z}}\right)=H^{1}\left(\mathbb{C}^{3} \backslash \mathbb{C}^{2}, \Theta_{\tilde{Z}}\right)=0$ as $H^{1}\left(\mathbb{C}^{3} \backslash \mathbb{C}^{2}, \mathcal{O}\right)=0$ by the $\bar{\partial}$-Poincaré lemma.

Now, let us study the cohomology groups $H^{j}\left(\tau_{\mathcal{Z} \mathcal{S}}^{0}\right)$. Let $\mathcal{Z}^{\prime}, \mathcal{S}^{\prime}$ be the normalizations of $\mathcal{Z}, \mathcal{S}$ respectively and let also $Q^{\prime}$ denote the disjoint union of $Q_{1}$ and $Q_{2}$. Then we have the following sequence on $\mathcal{Z}$

$$
0 \rightarrow \tau_{\mathcal{Z} \mathcal{S}}^{0} \rightarrow q_{*} \Theta_{\mathcal{Z}^{\prime} \mathcal{S}^{\prime} Q^{\prime}} \rightarrow i_{*} \Theta_{Q C \bar{C}} \rightarrow 0
$$

where $q: \mathcal{Z}^{\prime} \rightarrow \mathcal{Z}$ is the identification map and $i: Q \hookrightarrow \mathcal{Z}$ is the inclusion.

Lemma 4.3. For all $j \geq 0$ we have:

$$
\begin{aligned}
H^{j}\left(\mathcal{Z}, q_{*} \Theta_{\mathcal{Z}^{\prime} \mathcal{S}^{\prime} Q^{\prime}}\right) & =H^{j}\left(\mathcal{Z}^{\prime}, \Theta_{\mathcal{Z}^{\prime} \mathcal{S}^{\prime} Q^{\prime}}\right) \\
& =H^{j}\left(\tilde{Z}_{1}, \Theta_{\tilde{Z}_{1} \tilde{S} Q_{1}}\right) \oplus H^{j}\left(\tilde{Z}_{2}, \Theta_{\tilde{Z}_{2} \tilde{D}_{2} \tilde{\bar{D}}_{2} Q_{2}}\right) .
\end{aligned}
$$


Proof. This follows from the Leray Spectral sequence if we can prove the vanishing of $R^{i} q_{*} \Theta_{\mathcal{Z}^{\prime} \mathcal{S}^{\prime} Q^{\prime}}, i \geq 1$, or equivalently we need the vanishing of $H^{i}\left(U_{1}, \Theta_{\tilde{Z}_{1} \tilde{S} Q_{1}}\right)$ and $H^{i}\left(U_{2}, \Theta_{\tilde{Z}_{2} \tilde{D}_{2} \tilde{\bar{D}}_{2} Q_{2}}\right), i \geq 1$ for small open sets $U_{k}, k=$ 1 , 2. On $U_{1}$, where $U_{1} \cap \tilde{S} \cap Q_{1}$ is non-empty, we have

$$
0 \rightarrow \Theta_{\tilde{Z}_{1} \tilde{S} Q_{1}} \stackrel{i}{\longrightarrow} \Theta_{\tilde{Z}_{1} \tilde{S}} \stackrel{\pi}{\longrightarrow} \mathcal{O}_{Q_{1}}\left(N_{Q_{1}}^{\tilde{Z}_{1}}\right) \longrightarrow 0
$$

which is exact by the transversality of $Q_{1}$ and $\tilde{S}$ : Choose coordinates $\left(x_{1}, x_{2}, x_{3}\right)$ such that $x_{1}=0$ defines $\tilde{S}$ and $x_{2}=0$ defines $Q_{1}$. Then on $U_{1} \pi=r \circ d x_{2}: \Theta_{\tilde{Z}_{1} \tilde{S}} \rightarrow \mathcal{O}_{Q_{1}}\left(N_{Q_{1}}^{\tilde{Z}_{1}}\right)$ where $r$ is the restriction onto $Q_{1}$ and the exactness is now easily seen. Then, from Lemma 4.1 and the sequence (4.2) we get $H^{i}\left(U_{1}, \Theta_{\tilde{Z}_{1} \tilde{S} Q_{1}}\right)=0, i \geq 2$ and as (4.2) is true on the level of presheafs we have also the vanishing for $i=1$. The vanishing of $H^{i}\left(U_{2}, \Theta_{\tilde{Z}_{2} \tilde{D}_{2} \tilde{\bar{D}}_{2} Q_{2}}\right), i \geq 1$, may be obtained as above by choosing $U_{2}$ so small that it meets only $\tilde{D}_{2}$ (or $\tilde{\bar{D}}_{2}$ ).

Lemma 4.4. For all $j \geq 0, H^{j}\left(\tilde{Z}_{1}, \Theta_{\tilde{Z}_{1} \tilde{S} Q_{1}}\right)=H^{j}\left(Z_{1}, \Theta_{Z_{1} S L_{1}}\right)$.

Proof. As in the proof of Lemma 4.2 we have $b_{1 *} \Theta_{\tilde{Z}_{1} \tilde{S} Q_{1}}=\Theta_{Z_{1} S L_{1}}$. The result follows from the Leray spectral sequence once we have established the vanishing of $R^{i} b_{1^{*}}\left(\Theta_{\tilde{Z}_{1} \tilde{S} Q_{1}}\right)$ for $i \geq 1$. We consider the sequences (4.2) and

$$
0 \rightarrow \Theta_{\tilde{Z}_{1} \tilde{S}} \rightarrow \Theta_{\tilde{Z}_{1}} \rightarrow \mathcal{O}_{\tilde{S}}\left(N_{\tilde{S}}^{\tilde{Z}_{1}}\right) \rightarrow 0 .
$$

Assume $\bar{q} \notin U \subseteq Z_{1}$ and that the bundles are trivial on $U$. Then on $b_{1}^{-1}(U)$ we have $\mathcal{O}_{\tilde{S}}\left(N_{\tilde{S}}^{\tilde{Z}_{1}}\right)=\left.[\tilde{S}]\right|_{\tilde{S}}=\left.\left.b_{1}^{*}[S]\right|_{\tilde{S}} \otimes Q_{1}^{-1}\right|_{\tilde{S}}=\left.b_{1}^{*}[S]\right|_{\tilde{S}} \otimes C_{1}^{-1}$. Thus on $b_{1}^{-1}(U) \cap \tilde{S}$

$$
0 \rightarrow \mathcal{O}_{\tilde{S}}\left(N_{\tilde{S}}^{\tilde{Z}_{1}}\right) \rightarrow b_{1}^{*}[S] \rightarrow \mathcal{O}_{C_{1}}\left(b_{1}^{*}[S]\right) \rightarrow 0
$$

is exact. Also, $H^{j}\left(b_{1}^{-1}(U) \cap C_{1}, b_{1}^{*}[S]\right)=H^{j}(q,[S])=0$ for $j \geq 1, H^{0}(q,[S])=$ $[S]_{q}=\mathbb{C}$ and $H^{j}\left(b_{1}^{-1}(U) \cap \tilde{S}, b_{1}^{*}[S]\right)=H^{j}(U \cap S,[S])=0$, for $j \geq 1$, by the $\bar{\partial}$-Poincaré Lemma. Thus, $H^{j}\left(b_{1}^{-1}(U) \cap \tilde{S}, \mathcal{O}_{\tilde{S}}\left(N_{\tilde{S}}^{\tilde{Z}_{1}}\right)\right)=0, j \geq 1$, if we can prove surjectivity of

$$
H^{0}\left(b_{1}^{-1}(U) \cap \tilde{S}, b_{1}^{*}[S]\right) \rightarrow H^{0}\left(b_{1}^{-1}(U) \cap C_{1}, b_{1}^{*}[S]\right) .
$$

This corresponds via $b_{1}$ to the map $H^{0}(U \cap S,[S]) \rightarrow H^{0}(q,[S])$ which is onto by evaluation. From the proof of Lemma 4.2 we also have $H^{j}\left(b_{1}^{-1}(U), \Theta_{\tilde{Z}_{1}}\right)=$ $0, j \geq 1$. Thus, from $(4.3), H^{j}\left(b_{1}^{-1}(U), \Theta_{\tilde{Z}_{1} \tilde{S}}\right)=0, j \geq 1$, once we have proved that

$$
H^{0}\left(b_{1}^{-1}(U), \Theta_{\tilde{Z}_{1}}\right) \rightarrow H^{0}\left(b_{1}^{-1}(U) \cap \tilde{S}, \mathcal{O}_{\tilde{S}}\left(N_{\tilde{S}}^{\tilde{Z}_{1}}\right)\right)
$$


is onto. However, it is not hard to describe the vector fields on $b_{1}^{-1}(U)$ in local coordinates on patches $V_{1}, V_{2}$ as in the proof of Lemma 4.2 and to see that the map is surjective.

Now, as $H^{j}\left(b_{1}^{-1}(U), \mathcal{O}_{Q_{1}}\left(N_{Q_{1}}^{\tilde{Z}_{1}}\right)\right)=0, j \geq 1$, we get from (4.2) that $H^{j}\left(b_{1}^{-1}(U), \Theta_{\tilde{Z}_{1} \tilde{S} Q_{1}}\right)=0, j \geq 2$. The map

$$
H^{0}\left(b_{1}^{-1}(U), \Theta_{\tilde{Z}_{1} \tilde{S}}\right) \rightarrow H^{0}\left(b_{1}^{-1}(U), \mathcal{O}_{Q_{1}}\left(N_{Q_{1}}^{\tilde{Z}_{1}}\right)\right)
$$

is onto which again is seen using local coordinates on $b_{1}^{-1}(U)$. Thus we have $R^{i} b_{*}\left(\Theta_{\tilde{Z}_{1} \tilde{S} Q_{1}}\right)=0, i \geq 1$ and the lemma is proved.

Similarly we have:

Lemma 4.5. For all $j \geq 0, H^{j}\left(\tilde{Z}_{2}, \Theta_{\tilde{Z}_{2} \tilde{D}_{2} \tilde{\bar{D}}_{2} Q_{2}}\right)=H^{j}\left(Z_{2}, \Theta_{Z_{2} D_{2} \bar{D}_{2}}\right)$.

Proof. Again $b_{2^{*}} \Theta_{\tilde{Z}_{2} \tilde{D}_{2} \tilde{\bar{D}}_{2} Q_{2}}=\Theta_{Z_{2} D_{2} \bar{D}_{2}}$ via the differential $d b_{2}$. Furthermore the sheaf $R^{i} b_{2^{*}}\left(\Theta_{\tilde{Z}_{2} \tilde{D}_{2} \tilde{\bar{D}}_{2} Q_{2}}\right)$ vanishes for $i \geq 1$ : First we prove the vanishing of $H^{j}\left(b_{2}^{-1}\left(U_{2}\right), \Theta_{\tilde{Z}_{2} \tilde{D}_{2} \tilde{\bar{D}}_{2}}\right), j \geq 1, U_{2} \subseteq Z_{2}$. Use coordinates $\left(z_{1}, z_{2}, \ell\right)$, $\left(z_{1}, z_{3}, \frac{1}{\ell}\right)$ on a cover $V_{1} \cup V_{2}$ of $b_{2}^{-1}\left(U_{2}\right)$ such that $D$ is given by $z_{2}=0$, $\bar{D}$ by $z_{3}=0$ and $L_{2}$ by $z_{2}=z_{3}=0$. Lemma 4.1 gives that this is a Leray cover of $b_{2}^{-1}\left(U_{2}\right)$ because $\Theta_{\tilde{Z}_{2} \tilde{D}_{2} \tilde{\bar{D}}_{2}}\left(V_{2}\right)=\Theta_{\tilde{Z}_{2} \tilde{D}_{2}}\left(V_{2}\right)$ and $\tilde{D}_{2}$ in $V_{2}$ is a smooth hypersurface. Also, $\Theta_{\tilde{Z}_{2} \tilde{D}_{2} \tilde{\bar{D}}_{2}}\left(V_{1} \cap V_{2}\right)=\Theta_{\tilde{Z}_{2}}\left(\mathbb{C}^{3} \backslash \mathbb{C}^{2}\right)$. Therefore $H^{j}\left(b_{2}^{-1}\left(U_{2}\right), \Theta_{\tilde{Z}_{2} \tilde{D}_{2} \tilde{\bar{D}}_{2}}\right)$ vanishes, $j \geq 1$. Then consider the sequence

$$
0 \rightarrow \Theta_{\tilde{Z}_{2} \tilde{D}_{2} \tilde{\bar{D}}_{2} Q_{2}} \rightarrow \Theta_{\tilde{Z}_{2} \tilde{D}_{2} \tilde{\bar{D}}_{2}} \rightarrow \mathcal{O}_{Q_{2}}\left(N_{Q_{2}}^{\tilde{Z}_{2}}\right) \rightarrow 0 .
$$

The vanishing of $H^{j}\left(b_{2}^{-1}\left(U_{2}\right) \cap Q_{2}, \mathcal{O}_{Q_{2}}\left(N_{Q_{2}}^{\tilde{Z}_{2}}\right)\right), j \geq 1$ is seen using the Leray cover $V_{1}, V_{2}$. Also, using coordinates on $V_{1}, V_{2}$ we can show that vector fields may be lifted so we have surjectivity of

$$
H^{0}\left(b_{2}^{-1}\left(U_{2}\right), \Theta_{\tilde{Z}_{2} \tilde{D}_{2} \tilde{\bar{D}}_{2}}\right) \rightarrow H^{0}\left(b_{2}^{-1}\left(U_{2}\right), \mathcal{O}_{Q_{2}}\left(N_{Q_{2}}^{\tilde{Z}_{2}}\right)\right) .
$$

Then the short exact sequence gives $H^{j}\left(b_{2}^{-1}\left(U_{2}\right), \Theta_{\tilde{Z}_{2} \tilde{D}_{2} \tilde{\bar{D}}_{2} Q_{2}}\right)=0, j \geq 1$, and we have proved the lemma.

To make use of the sequence (4.1) we prove the following lemma.

Lemma 4.6. The cohomology groups $H^{j}\left(Q, \Theta_{Q C \bar{C}}\right)$ vanishes for $j \geq 1$ and $H^{0}\left(Q, \Theta_{Q C \bar{C}}\right)=s u\left(V^{+}\right) \oplus u(1)$.

Proof. $H^{j}\left(Q, \Theta_{Q}\right)=0, j \geq 1$ and $H^{0}\left(Q, \Theta_{Q}\right)=H^{0}\left(P\left(V_{x_{1}}^{+}\right), \mathcal{O}(2)\right) \oplus$ $H^{0}\left(P\left(V_{x_{1}}^{-}\right), \mathcal{O}(2)\right)=S^{2}\left(V_{x_{1}}^{+}\right)^{*} \oplus S^{2}\left(V_{x_{1}}^{-}\right)^{*}=s u\left(V_{x_{1}}^{-}\right) \oplus s u\left(V_{x_{1}}^{-}\right)$. From the sequence

$$
0 \rightarrow \Theta_{Q C \bar{C}} \rightarrow \Theta_{Q} \rightarrow \mathcal{O}_{C \bar{C}}\left(N_{C \bar{C}}^{Q}\right) \rightarrow 0
$$


and the vanishing of $H^{j}\left(C, \mathcal{O}_{C}\left(N_{C}^{Q}\right)\right), j \geq 1$ we get $H^{2}\left(Q, \Theta_{Q C \bar{C}}\right)=0$. Now, $H^{0}\left(Q, \Theta_{Q}\right)$ is generated by global $S U(2) \times S U(2)$ holomorphic transformations. Elements in $H^{0}\left(Q, \Theta_{Q C \bar{C}}\right)$ are the vector fields generated by actions leaving $C$ and $\bar{C}$ invariant, i.e. by rotations on $L_{1}$ leaving $q$ and $\bar{q}$ fixed. Thus $H^{0}\left(Q, Q_{C \bar{C}}\right)$ is generated by $S U\left(V_{x_{1}}^{+}\right) \times U(1)$ where $U(1) \leq S U\left(V_{x_{1}}^{-}\right)$. Also, the map

$$
H^{0}\left(Q, \Theta_{Q}\right) \rightarrow H^{0}\left(C, \mathcal{O}_{C}\left(N_{C}^{Q}\right)\right) \oplus H^{0}\left(\bar{C}, \mathcal{O}_{\bar{C}}\left(N_{\bar{C}}^{Q}\right)\right)
$$

is surjective so $H^{1}\left(Q, \Theta_{Q C \bar{C}}\right)=0$.

We are now able to prove the vanishing of the relative obstruction group. Proposition 4.7. Assume $H^{2}\left(Z_{1}, \Theta_{Z_{1} S}\right)=0$ and $H^{2}\left(Z_{2}, \Theta_{Z_{2} D_{2} \bar{D}_{2}}\right)=0$. Then the obstruction group $H^{2}\left(\tau_{\mathcal{Z}}^{0}\right)$ vanishes.

Proof. The long exact sequence associated to (4.1) together with Lemmae $4.3,4.4,4.5$ and 4.6 gives

$$
H^{2}\left(\mathcal{Z}, \tau_{\mathcal{Z S}}^{0}\right)=H^{2}\left(Z_{1}, \Theta_{Z_{1} S L_{1}}\right) \oplus H^{2}\left(Z_{2}, \Theta_{Z_{2} D_{2} \bar{D}_{2}}\right) .
$$

The second summand vanishes due to the assumption. Consider the exact sequence

$$
0 \rightarrow \Theta_{Z_{1} S L_{1}} \rightarrow \Theta_{Z_{1} S} \rightarrow \mathcal{O}_{L_{1}}\left(N_{L_{1}}^{Z_{1}}\right) \rightarrow 0 .
$$

We have $H^{1}\left(L_{1}, \mathcal{O}_{L_{1}}\left(N_{L_{1}}^{Z_{1}}\right)\right)=0$ and also $H^{2}\left(Z_{1}, \Theta_{Z_{1} S}\right)=0$ so the first summand also vanishes.

As explained in Section 2 we also need the following vanishing results.

Proposition 4.8. Assume $H^{2}\left(Z_{i}, \Theta_{Z_{i}}\right)=0, i=1,2, H^{2}\left(S, \Theta_{S}\right)=0$ and $H^{2}\left(D_{2}, \Theta_{D_{2}}\right)=0$. Then the obstruction groups $H^{2}\left(\tau_{\mathcal{Z}}^{0}\right)$ and $H^{2}\left(\tau_{\mathcal{S}}^{0}\right)$ vanishes.

Proof. Consider the normalization in the following sequence

$$
0 \rightarrow \tau_{\mathcal{Z}}^{0} \rightarrow q_{*} \Theta_{\mathcal{Z}^{\prime} Q^{\prime}} \rightarrow i_{*} \Theta_{Q} \rightarrow 0 .
$$

Then, from Lemma 4.1 and the Leray spectral sequence, we get

$$
H^{2}\left(\mathcal{Z}, q_{*} \Theta_{\mathcal{Z}^{\prime} Q^{\prime}}\right)=H^{2}\left(\tilde{Z}_{1}, \Theta_{\tilde{Z}_{1} Q_{1}}\right) \oplus H^{2}\left(\tilde{Z}_{2}, \Theta_{\tilde{Z}_{2} Q_{2}}\right) .
$$

From the sequence

$$
0 \rightarrow \Theta_{\tilde{Z}_{i} Q_{i}} \rightarrow \Theta_{\tilde{Z}_{i}} \rightarrow \mathcal{O}_{Q_{i}}\left(N_{Q_{i}}^{\tilde{Z}_{i}}\right) \rightarrow 0
$$

and the vanishing of $H^{j}\left(Q_{i}, \mathcal{O}_{Q_{i}}\left(N_{Q_{i}}^{\tilde{Z}_{i}}\right)\right), j \geq 1$, we have $H^{2}\left(\tilde{Z}_{i}, \Theta_{\tilde{Z}_{i} Q_{i}}\right)=$ $H^{2}\left(\tilde{Z}_{i}, \Theta_{\tilde{Z}_{i}}\right)$ which is equal to $H^{2}\left(Z_{i}, \Theta_{Z_{i} L_{i}}\right)$ by Lemma 4.2. Since $H^{j}\left(L_{i}, \mathcal{O}_{L_{i}}\left(N_{L_{i}}^{Z_{i}}\right)\right)=0, j \geq 1$, we have $H^{2}\left(Z_{i}, \Theta_{Z_{i} L_{i}}\right)=H^{2}\left(Z_{i}, \Theta_{Z_{i}}\right)$, $i=1,2$, and since $H^{j}\left(Q_{i}, \Theta_{Q_{i}}\right)=0, i=1,2, j \geq 1$, the long exact sequence 
associated to (4.6) gives $H^{2}\left(\tau_{\mathcal{Z}}^{0}\right)=H^{2}\left(Z_{i}, \Theta_{Z_{1}}\right) \oplus H^{2}\left(Z_{2}, \Theta_{Z_{2}}\right)$ which vanishes by assumptions. This was just a repetition of the result of Donaldson and Friedman [2].

Next we concentrate on the divisor. Again we have a normalization sequence

$$
0 \rightarrow \tau_{\mathcal{S}}^{0} \rightarrow q_{*} \Theta_{\mathcal{S}^{\prime} C^{\prime} \bar{C}^{\prime}} \rightarrow i_{*} \Theta_{C \bar{C}} \rightarrow 0 .
$$

Lemma 4.1 together with the Leray spectral sequence give

$$
H^{2}\left(\mathcal{S}, q_{*} \Theta_{\mathcal{S}^{\prime} C^{\prime} \bar{C}^{\prime}}\right)=H^{2}\left(\tilde{S}, \Theta_{\tilde{S} C_{1} \bar{C}_{1}}\right) \oplus H^{2}\left(\tilde{D}_{2}, \Theta_{\tilde{D}_{2} C_{2}}\right) \oplus H^{2}\left(\tilde{\bar{D}}_{2}, \Theta_{\tilde{\bar{D}}_{2} \bar{C}_{2}}\right) \text {. }
$$

Then, as $H^{j}\left(C_{1}, \mathcal{O}_{C_{1}}\left(N_{C_{1}}^{\tilde{S}}\right)\right)=H^{j}\left(\mathbb{C P}^{1}, \mathcal{O}(-1)\right)=0, j \geq 0$, the sequence

$$
0 \rightarrow \Theta_{\tilde{S} C_{1} \bar{C}_{1}} \rightarrow \Theta_{\tilde{S}} \rightarrow \mathcal{O}_{C_{1}}\left(N_{C_{1}}^{\tilde{S}}\right) \oplus \mathcal{O}_{\bar{C}_{1}}\left(N_{\bar{C}_{1}}^{\tilde{S}}\right) \rightarrow 0
$$

gives $H^{j}\left(\tilde{S}, \Theta_{\tilde{S} C_{1} \bar{C}_{1}}\right)=H^{j}\left(\tilde{S}, \Theta_{\tilde{S}}\right), j \geq 0$. Essentially by repeating the arguments in Lemma 4.2 we get $H^{j}\left(\tilde{S}, \Theta_{\tilde{S}}\right)=H^{j}\left(S, \Theta_{S q \bar{q}}\right), j \geq 0$. For dimensional reasons we have $H^{j}\left(q \bar{q}, \mathcal{O}_{q \bar{q}}\left(N_{q \bar{q}}^{S}\right)\right)=0, j \geq 1$, so $H^{2}\left(S, \Theta_{S q \bar{q}}\right)=$ $H^{2}\left(S, \Theta_{S}\right)$. For $\tilde{D}_{2}$ we have $N_{C_{2}}^{\tilde{D}} \cong \mathcal{O}(1)$ so the sequence

$$
0 \rightarrow \Theta_{\tilde{D}_{2} C_{2}} \rightarrow \Theta_{\tilde{D}_{2}} \rightarrow \mathcal{O}_{C_{2}}\left(N_{C_{2}}^{\tilde{D}_{2}}\right) \rightarrow 0
$$

gives $H^{2}\left(\tilde{D}_{2}, \Theta_{\tilde{D} C_{2}}\right)=H^{2}\left(\tilde{D}_{2}, \Theta_{\tilde{D}_{2}}\right)$. Then as $\Theta_{C_{2}}=\mathcal{O}(2)$ the long exact sequence associated to (4.7) gives

$$
H^{2}\left(\tau_{\mathcal{S}}^{0}\right)=H^{2}\left(S, \Theta_{S}\right) \oplus H^{2}\left(D_{2}, \Theta_{D_{2}}\right) \oplus H^{2}\left(\bar{D}_{2}, \Theta_{\bar{D}_{2}}\right)
$$

which vanishes by assumptions.

This ends our proof of the fact that under the general Assumption 2.1, the twistor space of the connected sum $M_{1} \# M_{2}$ has an irreducible degree 2 divisor.

Finally we want to summarize and at the same time bring the symmetries back into considerations.

Theorem 4.9. Let $M_{1}, M_{2}$ be compact self-dual conformal 4-manifolds with torus symmetry and fixed points. Assume the isotropy representations at the fixed points are intertwined via an orientation reversing isometry. If the Assumption 2.1 is satisfied for $T^{2}$-invariant divisors $S, D_{2}$ and $\bar{D}_{2}$, then there is a complex equivariant smoothing of the singular twistor space $\mathcal{Z}$ and the singular divisor $\mathcal{S}$ into a twistor space with torus action and an invariant irreducible degree 2 divisor.

Proof. Due to the equivariance of the various sheaf morphisms we get an analogue of diagram (2.7) where the morphisms are betweeen the $G$-invariant part of the groups. Consider the vanishing results in Propositions 4.7 and 4.8. The spectral sequence (2.1) now gives the vanishing of the obstruction spaces $T_{\mathcal{Z}}^{2}$ and $T_{\mathcal{S}}^{2}$. Then, chasing the diagram (2.7) gives surjectivity of the 
morphism $\gamma$ so $T_{f}^{2}=0$. Therefore, any element $\omega$ in the complement of the hyperplane $H^{1}\left(\tau_{\mathcal{Z} \mathcal{S}}^{0}\right)$ of $T_{f}^{1}$ gives smoothings of $\mathcal{Z}$ and $\mathcal{S}$ as $\omega$ maps to non-zero elements in $H^{0}\left(\tau_{\mathcal{S}}^{1}\right)$ and $H^{0}\left(\tau_{\mathcal{Z}}^{1}\right)$. Thus we obtain a smooth twistor space with an irreducible degree 2 divisor. Together with Lemma 3.1 this shows that there are $T^{2}$-equivariant deformations of $\mathcal{Z}$ and $\mathcal{S}$.

Remark. We can also prove $H^{2}\left(\tau_{\mathcal{Z} \mid \mathcal{S}}^{0}\right)=0$ if we assume that each component $M_{i}, i=1,2$, has positive scalar curvature. As this vanishing result is not used in this paper, we only give a short outline of the proof: Start with the normalization sequence

$$
0 \rightarrow \tau_{\mathcal{Z} \mid \mathcal{S}}^{0} \rightarrow q_{*} \Theta_{\mathcal{Z}^{\prime} Q^{\prime} \mid \mathcal{S}} \rightarrow i_{*} \Theta_{Q \mid C \bar{C}} \rightarrow 0 .
$$

Then, as $\left.\left(q_{*} \Theta_{\mathcal{Z}^{\prime} Q^{\prime}}\right)\right|_{\mathcal{S}}=q_{*}\left(\Theta_{\mathcal{Z}^{\prime} Q^{\prime} \mid \mathcal{S}^{\prime}}\right)$, we get

$$
\begin{aligned}
& H^{j}\left(\mathcal{S}, q_{*} \Theta_{\mathcal{Z}^{\prime} Q^{\prime} \mid \mathcal{S}}\right) \\
& =H^{j}\left(\tilde{S}, \Theta_{\tilde{Z}_{1} Q_{1} \mid \tilde{S}}\right) \oplus H^{j}\left(\tilde{D}_{2}, \Theta_{\tilde{Z}_{2} Q_{2} \mid \tilde{D}_{2}}\right) \oplus H^{j}\left(\tilde{\bar{D}}_{2}, \Theta_{\tilde{Z}_{2} Q_{2} \mid \tilde{\bar{D}}_{2}}\right), \quad j \geq 0 .
\end{aligned}
$$

We study each component in this sum. From the sequence

$$
0 \rightarrow \Theta_{\tilde{Z}_{1} Q_{1} \mid \tilde{S}} \rightarrow \Theta_{\tilde{Z}_{1} \mid \tilde{S}} \rightarrow \mathcal{O}_{C_{1} \bar{C}_{1}}\left(N_{Q_{1}}^{\tilde{Z}_{1}}\right) \rightarrow 0
$$

and the vanishing $H^{j}\left(\tilde{S}, \mathcal{O}_{C_{1} \bar{C}_{1}}\left(N_{Q_{1}}^{\tilde{Z}_{1}}\right)\right)=0, j \geq 0$, we get $H^{j}\left(\tilde{S}, \Theta_{\tilde{Z}_{1} Q_{1} \mid \tilde{S}}\right)=$ $H^{j}\left(\tilde{S}, \Theta_{\tilde{Z}_{1} \mid \tilde{S}}\right), j \geq 0$. Consider therefore the sequence

$$
0 \rightarrow \Theta_{\tilde{S}} \rightarrow \Theta_{\tilde{Z}_{1} \mid \tilde{S}} \rightarrow \mathcal{O}_{\tilde{S}}\left(N_{\tilde{S}}^{\tilde{Z}_{1}}\right) \rightarrow 0
$$

As $N_{\tilde{S}}^{\tilde{Z}_{1}}=\left.b_{1}^{*}[S]\right|_{\tilde{S}} \otimes\left[C_{1}\right]^{-1} \otimes\left[\bar{C}_{1}\right]^{-1}$ and $\mathcal{O}_{C_{1} \bar{C}_{1}}\left(b_{1}^{*}[S]\right)=\mathcal{O}_{C_{1} \bar{C}_{1}}$ we have

$$
\left.0 \rightarrow N_{\tilde{S}}^{\tilde{Z}_{1}} \rightarrow b_{1}^{*}[S]\right|_{\tilde{S}} \rightarrow \mathcal{O}_{C_{1} \bar{C}_{1}} \rightarrow 0 .
$$

Also, $H^{j}\left(\tilde{S}, b_{1}^{*}[S]\right)=H^{j}(S,[S])=H^{j}\left(S, K^{-\frac{1}{2}}\right), j \geq 0$. Then, due to the positive scalar curvature and the vanishing results of Hitchin [5], the sequence

$$
0 \rightarrow \mathcal{O}_{Z_{1}} \rightarrow K^{-\frac{1}{2}} \rightarrow \mathcal{O}_{S}\left(K^{-\frac{1}{2}}\right) \rightarrow 0
$$

gives $H^{2}\left(\tilde{S}, b_{1}^{*}[S]\right)=0$. Then $(4.11)$ gives $H^{2}\left(\tilde{S}, \mathcal{O}_{\tilde{S}}\left(N_{\tilde{S}}^{\tilde{Z}_{1}}\right)\right)=0$. From the sequence (4.10) and from $H^{2}\left(\tilde{S}, \Theta_{\tilde{S}}\right)=H^{2}\left(S, \Theta_{S q \bar{q}}\right)=H^{2}\left(S, \Theta_{S}\right)=0$ we get $H^{2}\left(\tilde{S}, \Theta_{\tilde{Z}_{1} \mid \tilde{S}}\right)=0$. Thus $H^{2}\left(\tilde{S}, \Theta_{\tilde{Z}_{1} Q_{1} \mid \tilde{S}}\right)=0$. To prove the vanishing of $H^{2}\left(\tilde{D}_{2}, \Theta_{\tilde{Z}_{2} Q_{2} \mid \tilde{D}_{2}}\right)$ we proceed as above with sequences similar to (4.9), (4.10), (4.11) and the use of vanishing results due to the positive scalar curvature of $M_{2}$. Thus we have $H^{2}\left(\mathcal{S}, q_{*} \Theta_{\mathcal{Z}^{\prime} Q^{\prime} \mid \mathcal{S}}\right)=0$ and (4.8) then gives $H^{2}\left(\mathcal{S}, \tau_{\mathcal{Z} \mid \mathcal{S}}^{0}\right)=0$. 


\section{A Local Moduli Space of Self-Dual Metrics on $n \mathbb{C P}^{2}$.}

We shall continue our study [13] of the local moduli of $T^{2}$-symmetric selfdual structures on the connected sums of $n$ copies of the complex projective plane. As in [13] it is assumed that the only orbits are tori on which $T^{2}$ acts freely, circles stabilized by some $S^{1}$-subgroup inside $T^{2}$ and isolated fixed points. We construct $n \mathbb{C P}^{2}$ from $\mathbb{C P}^{2}$ by attaching planes step by step and at the same time keeping divisors and symmetries. However, let us take the general approach a little further in the case of an irreducible degree 2 divisor.

From the equivariant version of (2.7) we get the sequence

$$
0 \rightarrow H^{1}\left(\tau_{\mathcal{Z S}}^{0}\right)_{T^{2}} \rightarrow\left(T_{f}^{1}\right)_{T^{2}} \rightarrow \Delta_{T^{2}} \rightarrow 0 .
$$

Similarly (4.1) and Lemmae 4.3, 4.4, 4.5 and 4.6 give the sequence

$$
\begin{aligned}
0 & \rightarrow H^{0}\left(\tau_{\mathcal{Z}}^{0}\right)_{T^{2}} \rightarrow H^{0}\left(Z_{1}, \Theta_{Z_{1} S L_{1}}\right)_{T^{2}} \oplus H^{0}\left(Z_{2}, \Theta_{Z_{2} D_{2} \bar{D}_{2}}\right)_{T^{2}} \\
& \rightarrow\left(s u\left(V^{+}\right) \oplus u(1)\right)_{T^{2}} \\
& \rightarrow H^{1}\left(\tau_{\mathcal{Z} \mathcal{S}}^{0}\right)_{T^{2}} \rightarrow H^{1}\left(Z_{1}, \Theta_{Z_{1} S L_{1}}\right)_{T^{2}} \oplus H^{1}\left(Z_{2}, \Theta_{Z_{2} D_{2} \bar{D}_{2}}\right)_{T^{2}} \rightarrow 0 .
\end{aligned}
$$

Furthermore, from (4.5) we have the sequence

$$
\begin{aligned}
0 & \rightarrow H^{0}\left(Z_{1}, \Theta_{Z_{1} S L_{1}}\right)_{T^{2}} \rightarrow H^{0}\left(Z_{1}, \Theta_{Z_{1} S}\right)_{T^{2}} \rightarrow H^{0}\left(L_{1}, \mathcal{O}\left(N_{L_{1}}^{Z_{1}}\right)\right)_{T^{2}} \\
& \rightarrow H^{1}\left(Z_{1}, \Theta_{Z_{1} S L_{1}}\right)_{T^{2}} \rightarrow H^{1}\left(Z_{1}, \Theta_{Z_{1} S}\right)_{T^{2}} \rightarrow 0 .
\end{aligned}
$$

Thus, putting (5.1), (5.2) and (5.3) together, we get a formula for the dimensions: Let $\chi_{1}\left(\mathcal{F}_{X}\right)_{T^{2}}=h^{0}\left(X, \mathcal{F}_{X}\right)_{T^{2}}-h^{1}\left(X, \mathcal{F}_{X}\right)_{T^{2}}$ for a sheaf $\mathcal{F}_{X}$ on a complex manifold $X$.

Proposition 5.1. Let $\left(T_{x_{1}} M_{1}\right)_{T^{2}}$ denote the real vector space of $T^{2}$-invariant tangent vectors at $x_{1}$ and let $C\left(T^{2}\right)$ denote the Lie algebra of the centralizer of $T^{2}$ in $S O(3) \times S O(3)$. Then

$$
\begin{aligned}
\operatorname{dim}\left(T_{f}^{1}\right)_{T^{2}}= & 1+h^{1}\left(\tau_{\mathcal{Z} \mathcal{S}}^{0}\right)_{T^{2}} \\
= & 1+h^{0}\left(\tau_{\mathcal{Z} \mathcal{S}}^{0}\right)_{T^{2}}+\operatorname{dim}_{\mathbb{R}}\left(T_{x_{1}} M_{1}\right)_{T^{2}}+\operatorname{dim}_{\mathbb{R}} C\left(T^{2}\right) \\
& -\chi_{1}\left(\Theta_{Z_{1} S}\right)_{T^{2}}-\chi_{1}\left(\Theta_{Z_{2} D_{2} \bar{D}_{2}}\right)_{T^{2}} .
\end{aligned}
$$

Proof. We have used the Kodaira equivalence (3.1) between sections of the normal bundle of the line $L_{1}$ in the twistor space and tangent vectors at the corresponding point $x_{1}$ in $M_{1}$. The symmetry group $T^{2}$ sits in $S O(3) \times S O(3)$ via the composition of the isotropy representation $i: T^{2} \rightarrow S O(4)$ and the representation of $S O(4)$ on $\Lambda_{+}^{2} \oplus \Lambda_{-}^{2}$.

Now we need a series of lemmae which will ensure that we have the general Assumption 2.1 satisfied and will make it possible to find the number $\operatorname{dim}\left(T_{f}^{1}\right)_{T^{2}}$ in the case of irreducible degree 2 divisors in the connected sums of complex projective planes. 
Lemma 5.2. Let $Z_{2}$ be the twistor space of $\mathbb{C P}^{2}$. Then $H^{2}\left(Z_{2}, \Theta_{Z_{2}}\right)=0$, $H^{2}\left(D_{2}, \Theta_{D_{2}}\right)=0, H^{2}\left(\bar{D}_{2}, \Theta_{\bar{D}_{2}}\right)=0, H^{0}\left(Z_{2}, \Theta_{Z_{2} D_{2} \bar{D}_{2}}\right) \cong(u(1) \oplus s u(2))_{\mathbb{C}} \subseteq$ $s u(3)_{\mathbb{C}}$ and $H^{1}\left(Z_{2}, \Theta_{Z_{2} D_{2} \bar{D}_{2}}\right)=H^{2}\left(Z_{2}, \Theta_{Z_{2} D_{2} \bar{D}_{2}}\right)=0$.

Proof. The space $H^{0}\left(Z_{2}, \Theta_{Z_{2}}\right)$ is generated by the complexifications $S U(3)_{\mathbb{C}}$ of the lifts of the isometries on $\mathbb{C P}^{2}$ to the flag

$$
Z_{2}=\left\{\left(\left[v_{0}, v_{1}, v_{2}\right],\left[\ell_{0}, \ell_{1}, \ell_{2}\right]\right) \mid \sum v_{i} \ell_{i}=0\right\} .
$$

Then $H^{0}\left(Z_{2}, \Theta_{Z_{2} D_{2} \bar{D}_{2}}\right)$ consists of the vector fields generated by the group that leaves $D_{2} \cup \bar{D}_{2}$ invariant. For $D_{2}$ given by $v_{0}=0$ and $\bar{D}_{2}$ by $\ell_{0}=0$ it is clear that $H^{0}\left(Z_{2}, \Theta_{Z_{2} D_{2} \bar{D}_{2}}\right)$ must contain and therefore be equal to the maximal subalgebra $(u(1) \oplus s u(2))_{\mathbb{C}}$ of $s u(3)_{\mathbb{C}}$. Since $Z_{2}$ is a flag manifold and $D_{2}$ is the blow-up of $\mathbb{C P}^{2}$ at one point, $H^{2}\left(Z_{2}, \Theta_{Z_{2}}\right)$ and $H^{2}\left(D_{2}, \Theta_{D_{2}}\right)$ vanish.

Lemma 4.5 and the vanishing of $H^{j}\left(Q_{2}, \mathcal{O}_{Q_{2}}\left(N_{Q_{2}}^{\tilde{Z}_{2}}\right)\right), j \geq 0$, give $H^{j}\left(Z_{2}\right.$, $\left.\Theta_{Z_{2} D_{2} \bar{D}_{2}}\right)=H^{j}\left(\tilde{Z}_{2}, \Theta_{\tilde{Z}_{2} \tilde{D}_{2} \tilde{\bar{D}}_{2} Q_{2}}\right)=H^{j}\left(\tilde{Z}_{2}, \Theta_{\tilde{Z}_{2} \tilde{D}_{2} \tilde{\bar{D}}_{2}}\right)$. Consider the sequence

$$
0 \rightarrow \Theta_{\tilde{Z}_{2} \tilde{D}_{2} \tilde{\bar{D}}_{2}} \rightarrow \Theta_{\tilde{Z}_{2}} \rightarrow \mathcal{O}_{\tilde{D}_{2} \tilde{\bar{D}}_{2}}\left(N_{\tilde{D}_{2} \tilde{\bar{D}}_{2}}^{\tilde{Z}_{2}}\right) \rightarrow 0 .
$$

Here $H^{j}\left(\tilde{Z}_{2}, \mathcal{O}_{\tilde{D}_{2} \tilde{\bar{D}}_{2}}\left(N_{\tilde{D}_{2} \tilde{\bar{D}}_{2}}^{\tilde{Z}_{2}}\right)\right)$ is equal to the sum of $H^{j}\left(D_{2},\left[D_{2}\right] \otimes\left[L_{2}\right]^{-1}\right)$ and the conjugate part. This follows because $N_{\tilde{D}_{2}}^{\tilde{Z}_{2}}=\left.b_{2}^{*}\left[D_{2}\right]\right|_{\tilde{D}_{2}} \otimes\left[C_{2}\right]^{-1}$ and $b_{2}$ gives isomorphisms $\tilde{D}_{2} \cong D_{2}, C_{2} \cong L_{2}$. Identify $D_{2}$ to the blowup of $\mathbb{C P}^{2}$ at one point with hyperplane class $H$ and exceptional divisor $E$, then $\left.D_{2}\right|_{D_{2}}=H-E$ and $\left.\bar{D}_{2}\right|_{D_{2}}=L_{2}=H[\mathbf{1 7}]$. It follows that $H^{j}\left(D_{2},\left[D_{2}\right] \otimes\left[L_{2}\right]^{-1}\right)$ vanishes for $j \geq 0$ as $H^{0}\left(D_{2},-E\right)=0, H^{0}\left(D_{2},-3 H+\right.$ $2 E)=0$ and $\chi(-E)=0$ by the Riemann-Roch formula. The sequence (5.5) now shows that $H^{j}\left(\tilde{Z}_{2}, \Theta_{\tilde{Z}_{2} \tilde{D}_{2} \tilde{\bar{D}}_{2}}\right)=H^{j}\left(\tilde{Z}_{2}, \Theta_{\tilde{Z}_{2}}\right)$ which coincide with $H^{j}\left(Z_{2}, \Theta_{Z_{2} L_{2}}\right)$ as shown in Lemma 4.2. Tracing these identities we now have $H^{j}\left(Z_{2}, \Theta_{Z_{2} D_{2} \bar{D}_{2}}\right)=H^{j}\left(Z_{2}, \Theta_{Z_{2} L_{2}}\right), j \geq 0$. As $H^{j}\left(L_{2}, \mathcal{O}_{L_{2}}\left(N_{L_{2}}^{Z_{2}}\right)\right)=0$, $j \geq 1$, the sequence

$$
0 \rightarrow \Theta_{Z_{2} L_{2}} \rightarrow \Theta_{Z_{2}} \rightarrow N_{L_{2}}^{Z_{2}} \rightarrow 0
$$

gives $H^{2}\left(Z_{2}, \Theta_{Z_{2} L_{2}}\right)=H^{2}\left(Z_{2}, \Theta_{Z_{2}}\right)$ which vanishes for the flag. Thus, $H^{2}\left(Z_{2}, \Theta_{Z_{2} D_{2} \bar{D}_{2}}\right)=0$ as claimed. Also, as $D_{2} \cap \bar{D}_{2}=L_{2}, H^{0}\left(Z_{2}, \Theta_{Z_{2} L_{2}}\right)$ contains $H^{0}\left(Z_{2}, \Theta_{Z_{2} D_{2} \bar{D}_{2}}\right)=(u(1) \oplus s u(2))_{\mathbb{C}}$, which is maximal, so $H^{0}\left(Z_{2}\right.$, $\left.\Theta_{Z_{2} L_{2}}\right)$ is 4-dimensional. Since $H^{0}\left(L_{2}, \mathcal{O}_{L_{2}}\left(N_{L_{2}}^{Z_{2}}\right)\right)$ is 4-dimensional so (5.6) gives $H^{1}\left(Z_{2}, \Theta_{Z_{2} L_{2}}\right)=H^{1}\left(Z_{2}, \Theta_{Z_{2}}\right)$ which vanishes for the flag. Thus we get $H^{1}\left(Z_{2}, \Theta_{Z_{2} D_{2} \bar{D}_{2}}\right)=0$ and the lemma has been proved.

Consider the twistor space (5.4) of $\mathbb{C P}^{2}$ with the action of $T^{2}$ given by

$$
\left(\left[v_{0} e^{i \Phi_{1}}, v_{1} e^{i \Phi_{2}}, v_{2} e^{-i\left(\Phi_{1}+\Phi_{2}\right)}\right],\left[\ell_{0} e^{-i \Phi_{1}}, \ell_{1} e^{-i \Phi_{2}}, \ell_{2} e^{i\left(\Phi_{1}+\Phi_{2}\right)}\right]\right)
$$


where $\Phi_{i}=p_{i} \theta+q_{i} \psi, i=1,2,(\theta, \psi) \in T^{2}$ and the integers $p_{i}, q_{i}$ satisfy $p_{1} q_{2}-$ $p_{2} q_{1}= \pm 1[\mathbf{1 3}]$. Then the irreducible degree 2 divisor $S$ given by $\sum \lambda_{i} v_{i} \ell_{i}=$ 0 , where $\lambda_{i} \neq \lambda_{j}, i \neq j$, is $T^{2}$-invariant. Indeed $T^{2}$ is exactly the symmetry group of $S$. The twistor fibration onto $\mathbb{C P}^{2}$ is $\left[z_{0}, z_{1}, z_{2}\right]=\left[v_{1} \bar{\ell}_{2}-v_{2} \bar{\ell}_{1}, v_{2} \bar{\ell}_{0}-\right.$ $\left.v_{0} \bar{\ell}_{2}, v_{0} \bar{\ell}_{1}-v_{1} \bar{\ell}_{0}\right]$ and it induces the action $\left[z_{0} e^{-i \Phi_{1}}, z_{1} e^{-i \Phi_{2}}, z_{2} e^{i\left(\Phi_{1}+\Phi_{2}\right)}\right]$ on $\mathbb{C P}^{2}$. Take $x=[0,0,1] \in \mathbb{C P}^{2}$. Then the twistor line $L_{x}$ is given by $v_{2}=$ $\ell_{2}=0$ with two fixed points $q=([0,1,0],[1,0,0])$ and $\bar{q}=([1,0,0],[0,1,0])$. A generic $T^{2}$-invariant degree 2 divisor is transversal to $L_{x}$ and contains $q$ and $\bar{q}$.

We get a reducible invariant divisor with $D_{1}$ given by $v_{0}=0$ and $\bar{D}_{1}$ given by $\ell_{0}=0$. We have $L_{x} \cap D_{1}=q$ and $L_{x} \cap \bar{D}_{1}=\bar{q}$. Finally take $D_{2}$ given by $v_{2}=0$ and $\bar{D}_{2}$ corresponding to $\ell_{2}=0$. Then $L_{x}=D_{2} \cap \bar{D}_{2}$. Thus we have the following building blocks.

Lemma 5.3. The twistor space $Z$ of $\mathbb{C P}^{2}$ contains $T^{2}$-invariant divisors $S$, $D_{i}, \bar{D}_{i}, i=1,2$ as in Section 2 with arbitrary isotropy representation at fixed points and satisfying $H^{0}\left(Z, \Theta_{Z S}\right)_{T^{2}}=\mathbb{C}^{2}, H^{2}\left(S, \Theta_{S}\right)=0, H^{2}\left(D_{i}, \Theta_{D_{i}}\right)=0$, $H^{2}\left(\bar{D}_{i}, \Theta_{\bar{D}_{i}}\right)=0$ and $H^{2}\left(Z, \Theta_{Z D_{i} \bar{D}_{i}}\right)=0$.

Proof. The vanishing of $H^{2}\left(S, \Theta_{S}\right)$ follows because $H^{2}\left(Z, \Theta_{Z}\right)=0$ and because $S$ is contained in $Z$ with positive normal bundle. $H^{0}\left(Z, \Theta_{Z S}\right)_{T^{2}}$ contains at least the algebra generated by $T^{2}$ as $S$ is invariant and for the generic $S$ described above where $\lambda_{i} \neq \lambda_{j}, i \neq j$, the symmetry group can at most be two dimensional. With Lemma 5.2 and the discussion above in mind, the lemma is proved.

Note that in order to fulfill all the vanishing conditions stated in Assumptions 2.1 and 2.2 we still need to prove $H^{2}\left(Z, \Theta_{Z S}\right)=0$ for the flag manifold $Z$. This could be done, mutatis mutandis, as for $\Theta_{Z D \bar{D}}$ in Lemma 5.2. However, as we focus on the $T^{2}$-equivariant situation we only prove the vanishing of the $T^{2}$-invariant part of this cohomology group. Indeed it follows from the next more general lemma.

Lemma 5.4. Let $Z_{1}$ be the twistor space of $n \mathbb{C P}^{2}$ and let $S \subseteq Z_{1}$ be an irreducible degree 2 divisor with canonical bundle $K_{S}$. Then $H^{0}\left(S, \mathcal{O}\left(K_{S}^{-1}\right)\right)_{T^{2}} \cong$ $\mathbb{C}, H^{1}\left(S, \mathcal{O}\left(K_{S}^{-1}\right)\right)_{T^{2}}=0$ and $H^{2}\left(Z_{1}, \Theta_{Z_{1} S}\right)_{T^{2}}=0$.

Proof. It is known [14] that $S$ is the blow-up $S_{n}$ of a real quadric $S_{0} n$ times in a pair of conjugate points. Let $b_{k}: S_{k} \rightarrow S_{k-1}, 1 \leq k \leq n$, be the blow-down map from the blow-up of $S_{0} k$ times to the blow-up $k-1$ times.

The torus action has only isolated fixed points on $S_{n}$ so the action on $S_{k}$, $1 \leq k \leq n$, induced by blowing down, also only has isolated fixed points. Therefore, the points $q_{k-1}, \bar{q}_{k-1}$ of blowing-up from $S_{k-1}$ to $S_{k}$ is at the intersection of invariant divisors. For topological reasons the action on $S_{0}$ has four isolated fixed points. 
Let $K_{k}=K_{S_{k}}$ and let $E_{k}, \bar{E}_{k}$ be the exceptional divisor of the blowing-up $b_{k}$. Then $K_{k}^{-1}=b_{k}^{*} K_{(k-1)}^{-1} \otimes E_{k}^{-1} \otimes \bar{E}_{k}^{-1}$ and

$$
0 \rightarrow \mathcal{O}\left(K_{k}^{-1}\right) \rightarrow \mathcal{O}\left(b_{k}^{*} K_{(k-1)}^{-1}\right) \rightarrow \mathcal{O}_{E_{k} \cup \bar{E}_{k}}\left(b_{k}^{*} K_{(k-1)}^{-1}\right) \rightarrow 0
$$

is exact and induces

$$
\begin{aligned}
0 & \rightarrow H^{0}\left(S_{k}, \mathcal{O}\left(K_{k}^{-1}\right)\right)_{T^{2}} \rightarrow H^{0}\left(S_{k}, \mathcal{O}\left(b_{k}^{*} K_{(k-1)}^{-1}\right)\right)_{T^{2}} \\
& \rightarrow H^{0}\left(E_{k} \cup \bar{E}_{k}, \mathcal{O}\left(b_{k}^{*} K_{(k-1)}^{-1}\right)\right)_{T^{2}} \\
& \rightarrow H^{1}\left(S_{k}, \mathcal{O}\left(K_{k}^{-1}\right)\right)_{T^{2}} \rightarrow H^{1}\left(S_{k}, \mathcal{O}\left(b_{k}^{*} K_{(k-1)}^{-1}\right)\right)_{T^{2}} \rightarrow 0 .
\end{aligned}
$$

Now, $H^{0}\left(E_{k} \cup \bar{E}_{k}, \mathcal{O}\left(b_{k}^{*} K_{(k-1)}^{-1}\right)\right)$ is isomorphic to $K_{(k-1), q_{k-1}}^{-1} \oplus K_{(k-1), \bar{q}_{k-1}}^{-1}$. Furthermore, the $T^{2}$-action on $H^{0}\left(E_{k} \cup \bar{E}_{k}, \mathcal{O}\left(b_{k}^{*} K_{(k-1)}^{-1}\right)\right)$ is given as nontrivial rotations on each factor: Let $q_{k-1} \in S_{k-1}$ and let $A, B$ be two invariant divisors such that $q_{k-1} \in A \cap B$ and such that in local coordinates $\left(z_{1}, z_{2}\right), A$ is given by $z_{2}=0$ and $B$ is given as $z_{1}=0$. As the $T^{2}$-action does not have 2-dimensional fixed point set but only isolated fixed points, we may assume the action on $T_{q_{k-1}} S_{q_{k-1}}=T_{q_{k-1}} A \oplus T_{q_{k-1}} B$ is given as $\operatorname{diag}\left(e^{i \Phi_{1}}, e^{i \Phi_{2}}\right)$. On $K_{k-1, q_{k-1}}^{-1}=\Lambda^{2} T_{q_{k-1}} S_{q_{k-1}}$ the action is $e^{i\left(\Phi_{1}+\Phi_{2}\right)}$. This action will not be trivial due to the assumption $p_{1} q_{2}-p_{2} q_{1}= \pm 1$. On the blow-up the action is $\left(e^{i \Phi_{1}}, e^{i\left(\Phi_{2}-\Phi_{1}\right)}\right)$ near the intersection of the exceptional divisor and the proper transform $\tilde{A}$ and near $\tilde{B}$ it is $\left(e^{i \Phi_{2}}, e^{i\left(\Phi_{1}-\Phi_{2}\right)}\right)$. Therefore the weights must be linear independent at all the points $q_{k-1}$.

Thus $H^{0}\left(E_{k} \cup \bar{E}_{k}, \mathcal{O}\left(b_{k}^{*} K_{(k-1)}^{-1}\right)\right)_{T^{2}}$ vanishes. By an inductive argument we get from (5.7) that $H^{j}\left(S, \mathcal{O}\left(K_{S}^{-1}\right)\right)_{T^{2}}=H^{j}\left(S_{0}, \mathcal{O}\left(K_{0}^{-1}\right)\right)_{T^{2}}, j=0,1$. As $S_{0}$ is a quadric surface we have $H^{1}\left(S_{0}, \mathcal{O}\left(K_{0}^{-1}\right)\right)=0$. Choose coordinates $\left(\left[s_{0}, s_{1}\right],\left[t_{0}, t_{1}\right]\right)$ on $S_{0}=\mathbb{C P}^{1} \times \mathbb{C P}^{1}$ such that the action on $S_{0}$ is $\left(\left[s_{0}, s_{1} e^{i \Phi_{1}}\right],\left[t_{0}, t_{1} e^{i \Phi_{2}}\right]\right)$. The nine monomials $\left(s_{0}^{2} t_{0}^{2}, \ldots, s_{1}^{2} t_{1}^{2}\right)$ of order four give a basis of $H^{0}\left(S_{0}, \mathcal{O}\left(K_{0}^{-1}\right)\right)=H^{0}\left(\mathbb{C P}^{1} \times \mathbb{C P}^{1}, \mathcal{O}(2,2)\right)$. The weights of the induced $T^{2}$-action vanishes on $s_{0}^{2} t_{0}^{2}$ and is of the form $n \Phi_{1}+m \Phi_{2}$ on the eight other monomials. We claim that $s_{0}^{2} t_{0}^{2}$ is the only invariant section: Note that if the weights $\left(\Phi_{1}, \Phi_{2}\right)$ of the isotropy representation at $([1,0],[1,0]) \in S_{0}$ are linearly dependent then the weights of the isotropy representations at the other three fixed points on $S_{0}$ are also linearly dependent. Since the weights at the fixed points of the $T^{2}$-action on the blow-up are linear combinations of $\Phi_{1}$ and $\Phi_{2}$ the weights on $S$ will be linearly dependent too. It follows, by restricting the twistor projection onto $S$, that on $n \mathbb{C P}^{2}$ there is a fixed point where $p_{1} q_{2}-p_{2} q_{2}=0$ which is a contradiction. Thus, $H^{0}\left(S, \mathcal{O}\left(K_{S}^{-1}\right)\right)_{T^{2}} \cong \mathbb{C}$. Finally, as $[S]=K_{S}^{-1}$, the sequence

$$
0 \rightarrow \Theta_{Z_{1} S} \rightarrow \Theta_{Z_{1}} \rightarrow \mathcal{O}_{S}\left(K_{S}^{-1}\right) \rightarrow 0
$$


gives $H^{2}\left(Z_{1}, \Theta_{Z_{1} S}\right)_{T^{2}}=0$.

We are now able to find the number $\operatorname{dim}\left(T_{f}^{1}\right)_{T^{2}}$ in a concrete example.

Proposition 5.5. It is possible to construct a $T^{2}$-symmetric twistor space of $n \mathbb{C P}^{2}$ with invariant irreducible degree 2 divisors by equivariant relative smoothings using $\mathbb{C P}^{2}$ as building blocks and proceeding step by step. Furthermore, in the final step we have $\operatorname{dim}\left(T_{f}^{1}\right)_{T^{2}}=h^{1}\left(Z, \Theta_{Z S}\right)_{T^{2}}=n$, where $Z$ is the smooth twistor space of $n \mathbb{C P}^{2}$.

Proof. The assumptions which give unobstructed equivariant relative smoothings of two planes are satisfied. This follows from Lemmae 5.2, 5.3 and 5.4. Using upper semi-continuity of the dimension of cohomology we may indeed proceed step by step to get $T^{2}$-symmetric twistor spaces of $n \mathbb{C P}^{2}$ with degree 2 irreducible divisors.

With the assumption, $p_{1} q_{2}-p_{2} q_{1}= \pm 1$, on the $T^{2}$-action we have $\operatorname{dim}_{\mathbb{R}}\left(T_{x_{1}} M_{1}\right)_{T_{2}}=0$ and $\operatorname{dim}_{\mathbb{R}} C\left(T^{2}\right)=2$. Also, from Lemma 5.2, we get $\chi_{1}\left(\Theta_{Z_{2} D_{2} \bar{D}_{2}}\right)_{T^{2}}=\operatorname{dim}(u(1) \oplus s u(2))_{T^{2}}=2$. Lemma 5.3 and upper semicontinuity gives $h^{0}\left(Z_{1}, \Theta_{Z_{1} S}\right)_{T^{2}}=h^{0}\left(\tau_{\mathcal{Z} \mathcal{S}}^{0}\right)_{T^{2}}=2$ in each step. From the sequence (5.8) and Lemma 5.4 we get

$$
0 \rightarrow H^{0}\left(S, \mathcal{O}\left(K_{S}^{-1}\right)\right)_{T^{2}} \rightarrow H^{1}\left(Z_{1}, \Theta_{Z_{1} S}\right)_{T^{2}} \rightarrow H^{1}\left(Z_{1}, \Theta_{Z_{1}}\right)_{T^{2}} \rightarrow 0
$$

and $h^{0}\left(S, \mathcal{O}\left(K_{S}^{-1}\right)\right)_{T^{2}}=1$. In [13] we got $h^{1}\left(Z_{1}, \Theta_{Z_{1}}\right)_{T^{2}}=n-2$ if $Z_{1}$ corresponds to $(n-1) \mathbb{C P}^{2}$, so $h^{1}\left(Z_{1}, \Theta_{Z_{1} S}\right)_{T^{2}}=n-1$. Now, putting all these data into the formula in Proposition 5.1, we get $\operatorname{dim}\left(T_{f}^{1}\right)_{T^{2}}=n$ which by upper semi-continuity coincide with $h^{1}\left(Z, \Theta_{Z S}\right)_{T^{2}}$ for the smooth twistor space $Z$ of $n \mathbb{C P}$ with smooth irreducible degree 2 divisor $S$.

Thus, we may summarize and formulate the following result concerning the local moduli space of self-dual structures on $n \mathbb{C P}^{2}$ constructed in [13].

Theorem 5.6. Consider the local moduli space of $T^{2}$-symmetric self-dual structures on $n \mathbb{C P}^{2}$ obtained by equivariant smoothings. The self-dual structures are all associated to $T^{2}$-symmetric twistor spaces $Z$ with invariant irreducible degree 2 divisors. In particular, the scalar curvature of each Yamabe metric is positive. For $n \geq 3$, the $T^{2}$-action can be chosen such that all $S^{1}$ subgroups are non-semi-free. The dimension of the local moduli is equal to $h^{1}\left(Z, \Theta_{Z}\right)_{T^{2}}=n-1$.

Proof. Note that we have ensured that all twistor spaces have symmetry group of dimension 2 so a local moduli space is well-defined.

To prove this theorem, we need to prove that the deformation is target stable [18]. By taking the second components of the maps from the first 
column to the second column in diagram (2.7), we have

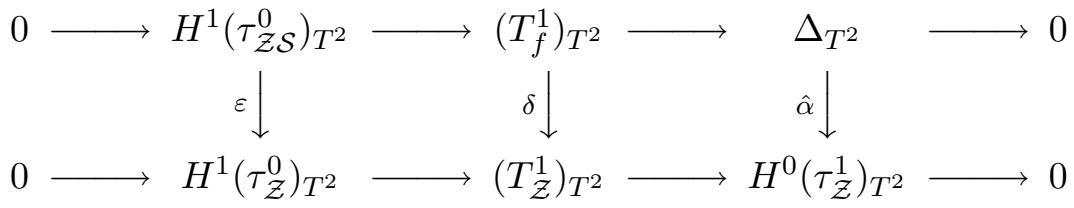

The target stability is equivalent to $\delta$ being surjective. Since $\hat{\alpha}$ is the identity map, $\delta$ is surjective if $\varepsilon$ is surjective.

By Lemma 5.3 and upper semi-continuity, the exact sequence (5.2) gives the isomorphism

$$
H^{1}\left(\tau_{\mathcal{Z S}}^{0}\right)_{T^{2}} \stackrel{\cong}{\longrightarrow} H^{1}\left(Z_{1}, \Theta_{Z_{1} S L_{1}}\right)_{T^{2}} .
$$

Also (5.3) gives the isomorphism

$$
H^{1}\left(Z_{1}, \Theta_{Z_{1} S L_{1}}\right)_{T^{2}} \stackrel{\cong}{\longrightarrow} H^{1}\left(Z_{1}, \Theta_{Z_{1} S}\right)_{T^{2}} .
$$

Similarly, one has the isomorphisms $[\mathbf{1 3}]$

$$
H^{1}\left(\tau_{\mathcal{Z}}^{0}\right)_{T^{2}} \stackrel{\cong}{\longrightarrow} H^{1}\left(Z_{1}, \Theta_{Z_{1} L_{1}}\right)_{T^{2}} \text { and } H^{1}\left(Z_{1}, \Theta_{Z_{1} L_{1}}\right)_{T^{2}} \stackrel{\cong}{\longrightarrow} H^{1}\left(Z_{1}, \Theta_{Z_{1}}\right)_{T^{2}} .
$$

Since the two compositions

$$
H^{1}\left(\tau_{\mathcal{Z}}^{0}\right)_{T^{2}} \stackrel{\cong}{\longrightarrow} H^{1}\left(Z_{1}, \Theta_{Z_{1} S}\right)_{T^{2}} \quad \text { and } \quad H^{1}\left(\tau_{\mathcal{Z}}^{0}\right)_{T^{2}} \stackrel{\cong}{\longrightarrow} H^{1}\left(Z_{1}, \Theta_{Z_{1}}\right)_{T^{2}}
$$

are induced by normalizations and restrictions, the inclusions induce a commutative diagram

$$
\begin{array}{ccc}
H^{1}\left(\tau_{\mathcal{Z} \mathcal{S}}^{0}\right)_{T^{2}} & \stackrel{\varepsilon}{\longrightarrow} & H^{1}\left(\tau_{\mathcal{Z}}^{0}\right)_{T^{2}} \\
H^{1}\left(Z_{1}, \Theta_{Z_{1} S}\right)_{T^{2}} & \stackrel{\lambda}{\longrightarrow} & H^{1}\left(Z_{1}, \Theta_{Z_{1}}\right)_{T^{2}} .
\end{array}
$$

By (5.9), $\lambda$ is surjective. Therefore $\varepsilon$ is surjective.

Thus, the local moduli space obtained in [13] does indeed correspond to twistor spaces carrying divisors as claimed. Therefore the corresponding Yamabe metrics are of non-negative type [3]. In fact the scalar curvature must be positive because the intersection form for $n \mathbb{C P}^{2}$ is positive definite [10]. The fact that we may assume the $S^{1}$ subgroups are all non-semi-free was proved in [13].

Turning to the problem of finding degree 1 divisors we first prove:

Lemma 5.7. If $L$ is the twistor line over a fixed point of a $T^{2}$-symmetric $n \mathbb{C P}^{2}$ and if $S$ is an invariant degree 2 divisor containing $L$, then $S$ is reducible. 
Proof. Assume on the contrary that $S$ is irreducible. Then $S$ is the blowup of a quadric surface with $L$ a smooth fiber [14]. In particular it does not pass through any points of blowing-up and this implies that $L$ is not invariant: On the quadric the only $T^{2}$-invariant curves are the two conjugate pair of generator lines passing through the four fixed points which are the only points of blowing-up as $S$ has $T^{2}$-symmetry.

Proposition 5.8. Given a $T^{2}$-symmetric twistor space over $n \mathbb{C P}^{2}$ with an invariant irreducible divisor $S$. Let $L$ be a twistor line above an isolated fixed point. Then there exists a conjugate pair $D, \bar{D}$ of invariant degree 1 divisors intersecting along $L$.

Proof. Let $V_{1}, V_{2}$ be a pair of independent holomorphic vector fields generated by the torus action on the twistor space $Z$. Since $S$ is invariant the restrictions of $V_{1}$ and $V_{2}$ to $S$ are tangential to $S$. Therefore we have an invariant section $\hat{s}=\left.V_{1} \wedge V_{2}\right|_{S} \in H^{0}\left(S, K_{S}^{-1}\right)$. We claim that this section is nontrivial. From Lemma $5.7 L$ intersects $S$ transversely at $q, \bar{q}$. Since the $T^{2}$-action only has isolated fixed points, there exist complex coordinates $\left(z_{1}, z_{2}\right)$ centered at $q$ such that the $T^{2}$-action is given as $\left(e^{i n \theta} z_{1}, e^{i m \psi} z_{2}\right)$ with $n$ and $m$ both non-zero. Therefore $\hat{s}=n m z_{1} z_{2} \frac{\partial}{\partial z_{1}} \wedge \frac{\partial}{\partial z_{2}}$ near $q$.

Since $H^{0}\left(S, K_{S}^{-1}\right)$ is non-trivial and $h^{1}(Z, \mathcal{O})=h^{1}\left(n \mathbb{C P}^{2}, \mathbb{R}\right)=0[5]$, the exact sequence

$$
0 \rightarrow \mathcal{O} \rightarrow K^{-\frac{1}{2}} \rightarrow \mathcal{O}_{S}\left(K_{S}^{-1}\right) \rightarrow 0
$$

implies that the section $\hat{s}$ lifts to an invariant section of $K^{-\frac{1}{2}}$.

Let $s \in H^{0}\left(Z, K^{-\frac{1}{2}}\right)$ be the section such that $s^{-1}(0)=S$. Then the subspace $\mathcal{V}=\operatorname{span}\{s, \hat{s}\}$ in $H^{0}\left(Z, K^{-\frac{1}{2}}\right)$ is $T^{2}$-invariant. Every element of this system contains $q$ and $\bar{q}$ and we may choose an element $S^{\prime} \in|\mathcal{V}|$ containing $p \in L$ where $p \neq q$ and $p \neq \bar{q}$. As $S^{\prime}$ is of degree 2 it also contains $L$. We may assume $S^{\prime}$ is real. The divisor $S^{\prime}$ is invariant, otherwise as $L$ is invariant, $L$ would be contained in the base locus of the system $|\mathcal{V}|$ but $S$ does not contain $L$. Then by Lemma $5.7, S^{\prime}$ must be reducible.

Corollary 5.9. The twistor spaces described in Theorem 5.6 contain $T^{2}$ invariant reducible degree 2 divisors.

Remark. Given a $T^{2}$-symmetric twistor space over $n \mathbb{C P}^{2}$ with an invariant reducible degree 2 divisor as above, we construct $T^{2}$-symmetric singular twistor spaces with degree 2 divisors over $(n+1) \mathbb{C P}^{2}$ as outlined at the end of Section 2. As in Section 4, mutatis mutandis, we can prove that the obstructions to the equivariant relative smoothing vanish provided Assumption 2.2 is satisfied. The cohomology group $H^{2}\left(Z_{i}, \Theta_{Z_{i}}\right)$ vanishes by upper semicontinuity. The groups $H^{2}\left(D_{i}, \Theta_{D_{i}}\right), H^{2}\left(\bar{D}_{i}, \Theta_{\bar{D}_{i}}\right), i=1,2 ; H^{2}\left(Z_{1}, \Theta_{Z_{1} S}\right)_{T^{2}}$ and $H^{2}\left(Z_{2}, \Theta_{Z_{2} D_{2} \bar{D}_{2}}\right)_{T^{2}}$ is proved to vanish as outlined at the end of the 
paper. However, as we do not control the singularity of the degree 2 divisors in the smoothing process, we cannot conclude a priori that there are reducible degree 2 divisors after the smoothing. Furthermore, the notion of local moduli becomes dubious when the degree 2 divisor is reducible. We do not pursue this issue. However, the existence of reducible degre 2 divisors is secured by Corollary 5.9. This has applications as shown in the next section.

\section{Anti-Self-Dual Hermitian Surfaces.}

In [13] we constructed self-dual metrics on $\left(S^{1} \times S^{3}\right) \# n \mathbb{C P}^{2}, n \geq 3$, such that the symmetry group is $S^{1}$ and the action is non-semi-free. We begin with a $\mathbb{C P}^{2}$ with $S^{1}$-action $\left[z_{0} e^{i \theta}, z_{1}, z_{2}\right]$. We label the fixed points as $P_{0}=[0,1,0]$, $P_{1}=[1,0,0]$ and $A_{1}=[0,0,1]$. Then we attach a $\mathbb{C P}^{2}$ with $S^{1}$-action $\left[z_{0} e^{-i \theta}, z_{1}, z_{2}\right]$ and fixed points $A_{2}=[0,0,1], P_{2}=[1,0,0], P_{\infty}=[0,1,0]$. We attach $A_{1}$ to $A_{2}$ via an orientation reversing isometry and of course using the smoothing of the twistor space. Note that the isotropy representation of $P_{0}$ is $\left(e^{i \theta} x, y\right)$ while near $P_{\infty}$ it is $\left(e^{-i \theta} x, y\right)$. Therefore, we may consider the possibility of making a self sum by identifying $P_{0}$ and $P_{\infty}$. Note that at this stage the $S^{1}$-action is semi-free but if we equivariantly attach a $\mathbb{C P}^{2}$ to $P_{2}$ we get a $S^{1}$-symmetric $3 \mathbb{C P}^{2}$ with non-semi-free action and we can still make a self-sum at $P_{0}$ and $P_{\infty}$. Indeed we can go on attaching more $\mathbb{C P}^{2}$-blocks away from $P_{0}$ and $P_{\infty}$ and then make a self-sum at $P_{0}$ and $P_{\infty}$ to obtain self-dual structures on $\left(S^{1} \times S^{3}\right) \# n \mathbb{C P}^{2}, n \geq 3$, with non-semi-free $S^{1}$-symmetry.

To get a complex structure on $\left(S^{1} \times S^{3}\right) \# n \mathbb{C P}^{2}$ compatible with the conformal metric but with opposite orientation we bring in the relative smoothing: Due to Corollary 5.9, we may assume that on $n \mathbb{C P}^{2}$ we have a non-semi-free $S^{1}$-symmetric twistor space with a reducible divisor $D+\bar{D}$ such that $D \cap \bar{D}=L_{P_{0}}$ and such that the twistor line above $P_{\infty}$ intersect $D$ and $\bar{D}$ transversely. Then make an equivariant self-sum at $P_{0}, P_{\infty}$ relative to the divisor $D+\bar{D}$. Following the notation from Section 2 we have curves $C_{1}, \bar{C}_{1}$ in $\tilde{D}, \tilde{\bar{D}}$ above $P_{\infty}$. Also, there are curves $C_{2}, \tilde{\bar{C}}_{2}$ above $P_{0}$ which are both mapped onto $L_{P_{0}}$ by the blowing-down.

Theorem 6.1. There exist anti-self-dual conformal Hermitian metrics on $\left(S^{1} \times S^{3}\right) \# n \overline{\mathbb{C P}}^{2}$, for $n \geq 3$, such that the symmetry group is $S^{1}$ and the action is non-semi-free.

Proof. We claim the isotropy data ensures that $C_{1}$ in $\tilde{D}$ is identified to $C_{2}$ in $\tilde{D}$ and not to $\bar{C}_{2}$ in $\tilde{\bar{D}}$ : On $2 \mathbb{C P}^{2}$ we have the four fixed points $P_{0}, P_{1}, P_{2}, P_{\infty}$. 
We draw the spheres between points as lines and get a diagram:

\begin{tabular}{c|cc|c}
$P_{\infty}$ & -1 & 1 & $P_{2}$ \\
\hline 0 & & & 1 \\
& & & \\
& & & \\
0 & & & -1 \\
\hline$P_{0}$ & 1 & -1 & $P_{1}$
\end{tabular}

Here the numbers represent the weights of the $S^{1}$-action along the spheres near the fixed points. These numbers are easily obtained from the information above about the $S^{1}$ action. The twistor space of $\mathbb{C P}^{2}$ was described in (5.4). We see that the twistor line $L_{P_{0}}$ is given by $v_{1}=0=\ell_{1}$ and the divisor corresponds to $v_{1}=0$ and its conjugate corresponds to $\ell_{1}=0$. On $L_{P_{0}}$ the $S^{1}$-action has the two fixed points $q_{0}=([0,0,1],[1,0,0])$ and $\bar{q}_{0}=([1,0,0],[0,0,1])$. On the divisor the isotropy data is given by

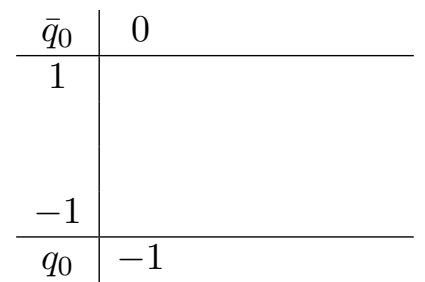

Now, the divisor $D$ in the twistor space of $2 \mathbb{C P}^{2}$ is the blow up of $\mathbb{C P}^{2}$ twice [17]. It has the following configuration

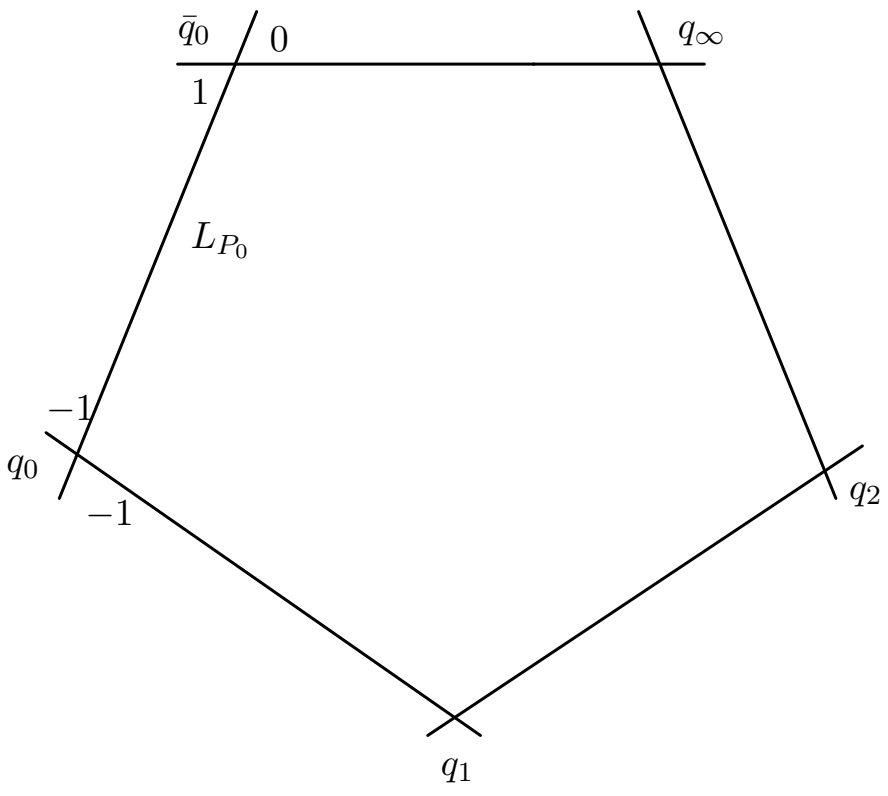


with $\pi\left(q_{i}\right)=p_{i}$ where $\pi$ is the restriction of the twistor projection

$$
\pi: D \rightarrow 2 \mathbb{C P}^{2} .
$$

Note that $\pi$ maps the whole line $L_{P_{0}}$ to $P_{0}$ and otherwise is an orientation reversing equivariant diffeomorphism. Therefore, by comparing with the data (6.1) on $2 \mathbb{C P}^{2}$, we obtain the full isotropy data on $D$ :

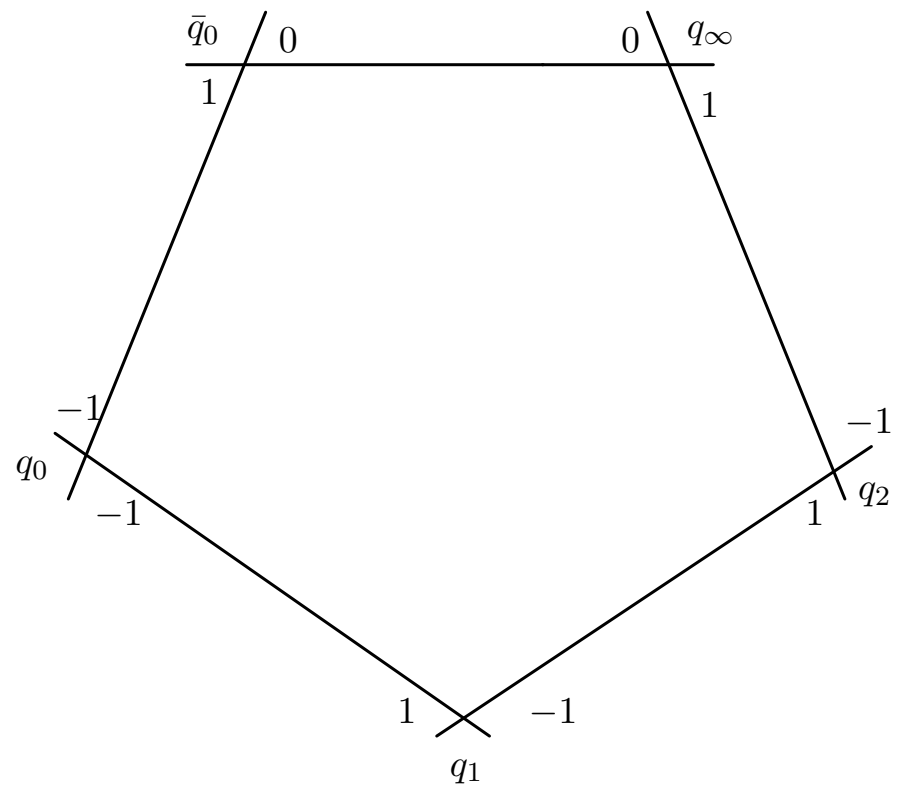

$(6.4)$

$q_{1}$

From the real structure $\sigma: D \rightarrow \bar{D}$ we can obtain the isotropy data on $\bar{D}$ near $q_{0}, \bar{q}_{0}, \bar{q}_{1}, \bar{q}_{2}, \bar{q}_{\infty}$. Then, as in the proof of Lemma 5.4 we get the data 
on the blow-up of $q_{\infty}$ : on $\tilde{D}$ we get

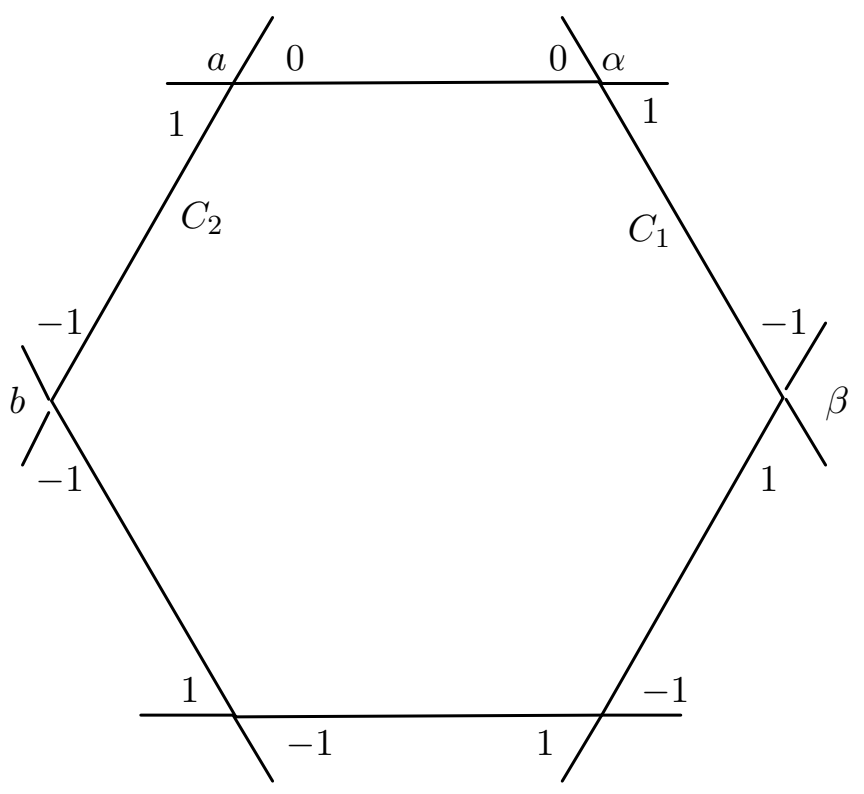

and on $\tilde{\bar{D}}$ we get

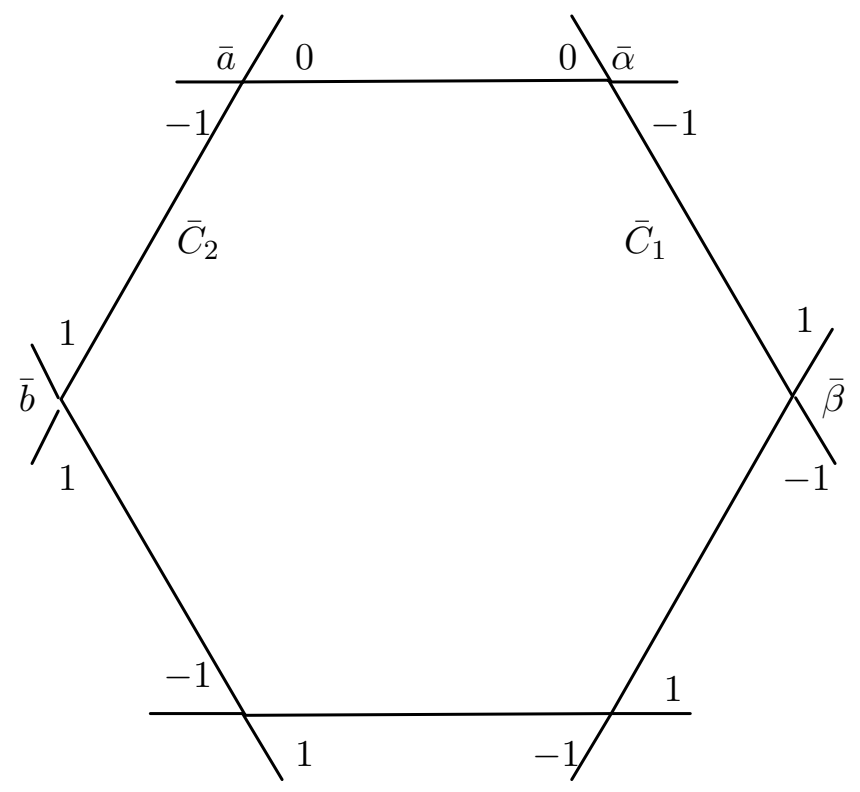

Here the points $a, \bar{b}$ are mapped to $\overline{q_{0}}$ by the blow-down and $\bar{a}, b$ are mapped to $q_{0}$. The points $\alpha, \bar{\alpha}$ corresponds to $q_{\infty}, \bar{q}_{\infty}$ while $\beta, \bar{\beta}$ correspond to $q_{2}, \overline{q_{2}}$. For the sake of completeness we may compute the isotropy data at $a, b, \alpha, \beta, \bar{a}, \bar{b}, \bar{\alpha}, \bar{\beta}$ also in directions transversal to the surfaces $\tilde{D}, \tilde{\bar{D}}$ : At 
each point let $(z, v, w)$ denote coordinates with $(v, w)$ coordinates along the exceptional divisor, the quadric surface, and with $(z, v)$ coordinates along the blown up divisor. Then with respect to such coordinates the weights of the isotropy is $a(0,1,1), b(-1,-1,1), \alpha(0,1,1), \beta(1,-1,1)$ and $\bar{a}(0,-1,-1), \bar{b}(1,1,-1), \bar{\alpha}(0,-1,-1), \bar{\beta}(-1,1,-1)$. The identification map $(z, v, w) \mapsto(\bar{z}, v, w)$ gives the orientation change on the divisor and it is now clear that $a$ is identified to $\alpha$, and $b$ to $\beta$.

This shows that we need to attach $C_{2}$ to $C_{1}$ and $\bar{C}_{2}$ to $\bar{C}_{1}$. For $n \mathbb{C P}^{2}$, in a neighbourhood of the invariant sphere joining the fixed points $P_{0}$ and $P_{\infty}$, the isotropy data is identical to the $2 \mathbb{C P}^{2}$ case. Therefore, the arguments above can be applied to prove the claim also for $n \mathbb{C P}^{2}$.

It follows that $\tilde{D}$ and $\tilde{\bar{D}}$ are glued to themselves respectively. Therefore, the singular divisor $\mathcal{S}$ in the singular twistor space $\mathcal{Z}$ is a disjoint union of two degree 1 divisors $\mathcal{D}$ and $\overline{\mathcal{D}}$. In the next paragraph, we prove that the obstructions to smoothing the pair $(\mathcal{Z}, \mathcal{S})$ vanish. The resulting smooth twistor space carries a conjugate pair of disjoint degree 1 divisors intersecting all twistor lines transversely. This pair corresponds to complex structures $\pm I$ on $\left(S^{1} \times S^{3}\right) \# n \mathbb{C P}^{2}$ compatible with the metric but inducing the opposite orientation.

To prove that the obstructions to the $S^{1}$-equivariant smoothing of the pair $(\mathcal{Z}, \mathcal{S})$ vanish, we proceed as in Section 4 . It suffices to prove the vanishing of $H^{2}\left(Z, \Theta_{Z D \bar{D}}\right)_{S^{1}}$ and $H^{2}\left(D, \Theta_{D}\right)_{S^{1}}$.

By Serre duality, $h^{2}\left(D, \Theta_{D}\right)=h^{0}\left(D, K_{D} \otimes \Omega^{1}\right)$. Since $D$ is $T^{2}$-invariant, $\left(V_{1} \wedge V_{2}\right)_{\mid D}$ is a non-trivial section of $K_{D}^{-1}$, where $V_{1}$ and $V_{2}$ is a pair of independent vector fields generated by the torus action on the twistor space. Therefore, if $h^{0}\left(D, K_{D} \otimes \Omega^{1}\right)$ was not equal to zero, there would have been non-trivial holomorphic 1 -forms on $D$. Since $D$ is a rational surface, it follows that $H^{2}\left(D, \Theta_{D}\right)$ vanishes.

To deal with $H^{2}\left(Z, \Theta_{Z D \bar{D}}\right)_{S^{1}}$, we consider the following exact sequences

$$
0 \rightarrow \Theta_{Z D \bar{D}} \rightarrow \Theta_{Z \bar{D}} \rightarrow \mathcal{O}_{D}\left(N_{D}^{Z}\right) \rightarrow 0,
$$

and

$$
0 \rightarrow \Theta_{Z \bar{D}} \rightarrow \Theta_{Z} \rightarrow \mathcal{O}_{\bar{D}}\left(N_{\bar{D}}^{Z}\right) \rightarrow 0 .
$$

By upper semi-continuity, $H^{2}\left(Z, \Theta_{Z}\right)$ vanishes. With the next lemma, we conclude that $H^{2}\left(Z, \Theta_{Z D \bar{D}}\right)_{S^{1}}$ vanishes.

Lemma 6.2. Let $Z$ be the twistor space of $n \mathbb{C P}^{2}$ with $T^{2}$ symmetry and let $D \subseteq Z$ be an invariant degree 1 divisor. Then $H^{j}\left(D, \mathcal{O}\left(N_{D}^{Z}\right)\right)_{S^{1}}=0$ and $H^{j}\left(\bar{D}, \mathcal{O}_{\bar{D}}\left(N_{\bar{D}}^{Z}\right)\right)=0, j \geq 1$.

Proof. Recall [17] that $D$ is the blow-up of $\mathbb{C P}^{2} n$ times. For $1 \leq k \leq n$ consider the sequence $b_{k}: D_{k} \rightarrow D_{k-1}$ of blowing down to $D_{0}=\mathbb{C P}^{2}$. Let $p_{k-1} \in D_{k-1}$ be the point which is blown up and let $E_{k}$ be the exceptional 
divisor. On $D=D_{n},\left[D_{n}\right]$ denotes the line bundle of $H-\sum_{i=1}^{n} E_{i}$ and on $D_{k},\left[D_{k}\right]$ is the line bundle of $H-\sum_{i=1}^{k} E_{i}[\mathbf{1 7}]$. Then $\left[D_{k}\right]=b_{k}^{*}\left[D_{k-1}\right] \otimes E_{k}^{-1}$ and from

$$
0 \rightarrow\left[D_{k}\right] \rightarrow b_{k}^{*}\left[D_{k-1}\right] \rightarrow \mathcal{O}_{E_{k}}\left(b_{k}^{*}\left[D_{k-1}\right]\right) \rightarrow 0
$$

we get

$$
\begin{aligned}
& \rightarrow H^{0}\left(D_{k},\left[D_{k}\right]\right)_{S^{1}} \rightarrow H^{0}\left(D_{k-1},\left[D_{k-1}\right]\right)_{S^{1}} \rightarrow\left(\left[D_{k-1}\right]_{p_{k-1}}\right)_{S^{1}} \\
& \rightarrow H^{1}\left(D_{k},\left[D_{k}\right]\right)_{S^{1}} \rightarrow H^{1}\left(D_{k-1},\left[D_{k-1}\right]\right)_{S^{1}} \rightarrow 0
\end{aligned}
$$

and $H^{2}\left(D_{k},\left[D_{k}\right]\right)=H^{2}\left(D_{k-1},\left[D_{k-1}\right]\right)$. Thus by induction we have

$$
H^{2}\left(D, \mathcal{O}\left(N_{D}^{Z_{1}}\right)\right)=H^{2}\left(D_{n},\left[D_{n}\right]\right)=H^{2}\left(D_{0},\left[D_{0}\right]\right)=H^{2}\left(\mathbb{C P}^{2},[H]\right)=0 .
$$

Now we prove $\left(\left[D_{k}\right]_{p_{k}}\right)_{S^{1}}=0,0 \leq k \leq n-1$. Let $g_{k}$ be the blow-down from $D_{k}$ to $\mathbb{C P}^{2}$. Then on $D_{k}$ we have $[\mathbf{1 7}]$

$$
K_{D_{k}}^{-1}=\left.\left.\left[\bar{D}_{k}\right]\right|_{D_{k}} \otimes\left[D_{k}\right]\right|_{D_{k}}=\left.\left(g_{k}^{*} H\right)^{2} \otimes\left[D_{k}\right]\right|_{D_{k}}
$$

so $\left(K_{D_{k}}^{-1}\right)_{p_{k}}=\left(g_{k}^{*} H\right)_{p_{k}}^{2} \otimes\left[D_{k}\right]_{p_{k}}$. In general, we may assume that the action on $\mathbb{C P}^{2}$ is $\left[z_{0}, z_{1} e^{i \Phi_{1}}, z_{2} e^{i \Phi_{2}}\right]$ in homogeneous coordinates. Then for $p=$ $g_{k}\left(p_{k}\right),\left(g_{k}^{*} H\right)_{p_{k}}^{3}=H_{p}^{3} \cong \Lambda^{2} T_{p} \mathbb{C P}^{2}$ so the weight of $\left(g_{k}^{*} H\right)_{p_{k}}^{2}$ is $\frac{2}{3}\left(\Phi_{1}+\Phi_{2}\right)$. Since $p_{k}$ is on the intersection of invariant curves $T_{p_{k}} D_{k}$ has weight $\left(n_{1} \Phi_{1}+\right.$ $\left.n_{2} \Phi_{2}, m_{1} \Phi_{1}+m_{2} \Phi_{2}\right)$. Then $\left(K_{D}^{-1}\right)_{p_{k}}$ has weight $m \Phi_{1}+n \Phi_{2}$ for some integers $m, n$. From diagram (6.4), after the curves joining $q_{1}$ to $q_{2}$, and $q_{2}$ to $q_{\infty}$ are blown down, we see that $\Phi_{1}=0$ and $\Phi_{2}=1$. Therefore, the representation on $\left[D_{k}\right]_{p_{k}}$ is non-trivial. This gives $\left(\left[D_{k}\right]_{p_{k}}\right)_{S^{1}}=0,0 \leq k \leq n-1$, and then by induction $(6.7)$ gives $H^{1}(D,[D])_{S^{1}}=0$.

Theorem 6.1 should be compared with LeBrun's examples [12] of $S^{1}$ symmetric anti-self-dual Hermitian metrics on blow-up of Hopf surfaces. Note that the $S^{1}$-action in his examples is semi-free.

\section{References}

[1] M.F. Atiyah, N.J. Hitchin and I.M. Singer, Self-duality in four-dimensional Riemannian geometry, Proc. Roy. Soc. London, A362 (1978), 425-461.

[2] S.K. Donaldson and R. Friedman, Connected sums of self-dual manifolds and deformations of singular spaces, Nonlinearity, 2 (1989), 197-239.

[3] P. Gauduchon, Structures de Weyl et théorèmes d'annulation sur une variété conforme autoduale, Norm. Sup. Pisa, 18 (1991), 563-629.

[4] R. Hartshorne, Algebraic Geometry, GTM 52, 1977, Springer-Verlag, New York.

[5] N.J. Hitchin, Linear field equations on self-dual spaces, Proc. Roy. Soc. London, Sér A, 370 (1980), 173-191.

[6] D. Joyce, Explicit construction of self-dual 4-manifolds, Duke Math. J., 77 (1995), 519-552. 
[7] J. Kim, On the scalar curvature of self-dual manifolds, Math. Ann., 297 (1993), 235-251.

[8] J. Kim and M. Pontecorvo, A new method of constructing scalar-flat Kähler surfaces, J. Differential Geometry, 41 (1995), 449-477.

[9] K. Kodaira and D. Spencer, On deformations of complex analytic structure, I, II, III, Ann. of Math., 67 (1958), 328-466; 71 (1960), 43-76.

[10] C. LeBrun, On the topology of self-dual 4-manifolds, Proc. Amer. Math. Soc., 98 (1986), 637-640.

[11] Explicit self-dual metrics on $\mathbb{C P}^{2} \# \cdots \# \mathbb{C P}^{2}$, J. Differential Geometry, 34 (1991), 223-253.

[12] _ Anti-self-dual Hermitian metrics on blown-up Hopf surfaces, Math. Ann., 289 (1991), 383-392.

[13] H. Pedersen and Y.S. Poon, Equivariant connected sums of compact self-dual manifolds, Math. Ann., 301 (1995), 717-749.

[14] - Self-duality and differentiable structures on the connected sum of complex projective planes, Proc. Amer. Math. Soc., 121 (1994), 859-864.

[15] Y.S. Poon, Compact self-dual manifolds with positive scalar curvature, J. Differential Geometry, 24 (1986), 97-132.

[16] _ Conformal transformations of compact self-dual manifolds, International J. Math., 5 (1994), 125-140.

[17] _ On the algebraic structure of twistor spaces, J. Differential Geometry, 36 (1992), 451-491.

[18] Z. Ran, Stability of certain holomorphic maps, J. Differential Geometry, 34 (1991), $37-47$.

[19] R. Schoen, Conformal deformation of a Riemannian metric to constant scalar curvature, J. Differential Geometry, 20 (1984), 478-495.

Received January 23, 1998. This project is partially supported by the NSF grant DMS9504908 .

ODENSE UNIVERSITY

DK-5230 ODEnSE M

DENMARK

E-mail address: henrik@imada.ou.dk

University of California, Riverside

Riverside, CA 92521

E-mail address: ypoon@math.ucr.edu 